\title{
Gaia Reveals a Metal-rich, in situ Component of the Local Stellar Halo
}

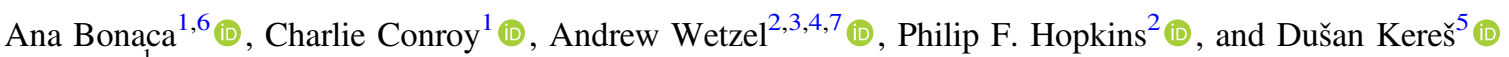 \\ ${ }^{1}$ Department of Astronomy, Harvard University, Cambridge, MA 02138, USA; ana.bonaca@cfa.harvard.edu \\ ${ }^{2}$ TAPIR, California Institute of Technology, Pasadena, CA, USA \\ 3 The Observatories of the Carnegie Institution for Science, Pasadena, CA, USA \\ ${ }^{4}$ Department of Physics, University of California, Davis, CA, USA \\ ${ }^{5}$ Department of Physics, Center for Astrophysics and Space Sciences, University of California, San Diego, La Jolla, CA, USA \\ Received 2017 April 17; revised 2017 June 29; accepted 2017 June 29; published 2017 August 16
}

\begin{abstract}
We use the first Gaia data release, combined with the RAVE and APOGEE spectroscopic surveys, to investigate the origin of halo stars within $\lesssim 3 \mathrm{kpc}$ from the Sun. We identify halo stars kinematically as moving at a relative speed of at least $220 \mathrm{~km} \mathrm{~s}^{-1}$ with respect to the local standard of rest. These stars are generally less metal-rich than the disk, but surprisingly, half of our halo sample is comprised of stars with $[\mathrm{Fe} / \mathrm{H}]>-1$. The orbital directions of these metal-rich halo stars are preferentially aligned with the disk rotation, in sharp contrast with the intrinsically isotropic orbital distribution of the metal-poor halo stars. We find similar properties in the Latte cosmological zoom-in simulation of a Milky Way-like galaxy from the FIRE project. In Latte, metal-rich halo stars formed primarily inside of the solar circle, whereas lower-metallicity halo stars preferentially formed at larger distances (extending beyond the virial radius). This suggests that metal-rich halo stars in the solar neighborhood actually formed in situ within the Galactic disk, rather than having been accreted from satellite systems. These stars, currently on halo-like orbits, therefore have likely undergone substantial radial migration/heating.
\end{abstract}

Key words: Galaxy: abundances - Galaxy: formation - Galaxy: halo - Galaxy: kinematics and dynamics - Galaxy: structure - solar neighborhood

\section{Introduction}

Early works suggested that the Galactic stellar halo formed in a dissipative collapse of a single protogalactic gas cloud (Eggen et al. 1962), but in today's prevalent cosmology, galaxies grow hierarchically by accreting smaller structure (White \& Rees 1978; Diemand et al. 2008; Springel et al. 2008; Klypin et al. 2011). The archaeological record of these galactic building blocks is especially well retained in stellar halos, partly because of their long relaxation times. Upon accretion, smaller galaxies completely disrupt within several orbital periods (Helmi \& White 1999) and become a part of the smooth halo. Still, global halo properties contain information on the original systems. For example, the total mass of a halo is related to the number of massive accretion events (Bullock \& Johnston 2005; Cooper et al. 2010), the outer slope of the outer halo density profile increases with the current accretion rate (Diemer \& Kravtsov 2014), and the presence of a break in the density profile is indicative of a quiet merger history at late times (Deason et al. 2013). Therefore, when studying the stellar halo of a galaxy, we are actually analyzing a whole population of accreted systems.

Modern works suggest that there are two distinct processes that contribute to the buildup of a stellar halo: most of the halo stars have been accreted from smaller galaxies, but a fraction of them have been formed in situ within the main galaxy (Zolotov et al. 2009; Font et al. 2011; Cooper et al. 2015). The in situ component can contain stars formed in the initial gas collapse (Samland \& Gerhard 2003) and/or stars formed in the disk, which have subsequently been kicked out and placed on halo orbits (Purcell et al. 2010). Throughout this work, we use the term in situ halo to address both of these origin scenarios.

\footnotetext{
${ }^{6}$ ITC Fellow.

7 Caltech-Carnegie Fellow.
}

Numerical simulations show that the total number of halo stars formed in situ depends on the details of the formation history, but their contribution generally decreases with distance from the galactic center (e.g., Zolotov et al. 2009; Cook et al. 2016). For Milky Way-like galaxies, $10 \%-30 \%$ of halo stars in the solar neighborhood are expected to have formed in situ (Zolotov et al. 2009). Thorough understanding of stellar halos relies on disentangling its accreted and in situ components.

Recent accretion events are still coherent in configuration space, and have been observed throughout the local universe. The first evidence of accretion came from studies of globular clusters (Searle \& Zinn 1978), but in recent years, deep photometric surveys on large scales have revealed a plethora of systems undergoing tidal disruption in the Milky Way halo, with progenitors ranging from dwarf galaxies (e.g., Ibata et al. 1994; Belokurov et al. 2007a, 2007b; Jurić et al. 2008; Bonaca et al. 2012b) to disrupted globular clusters (e.g., Rockosi et al. 2002; Grillmair \& Dionatos 2006; Grillmair 2009; Bonaca et al. 2012a; Bernard et al. 2016). A complete list of tidal structures identified in the Milky Way has recently been compiled by Grillmair \& Carlin (2016). Extragalactic surveys of low surface brightness features indicate that ongoing accretion is common in present-day galaxies of all masses (Ibata et al. 2001; Ferguson et al. 2002; MartínezDelgado et al. 2010, 2012; Romanowsky et al. 2012; Crnojević et al. 2016).

Evidence of past accretion events remains even after they no longer stand out as overdensities in configuration space. For example, Bell et al. (2008) measured that the spatial distribution of halo stars in the Milky Way is highly structured, to the degree expected by models where the entire halo was formed by accretion of dwarf galaxies. Expanding this approach to clustering in the space of both positions and radial velocities, Janesh et al. (2016) found that the amount of halo structure 
grows with distance from the Galactic center. However, phasespace substructure associated with mergers has been discovered even in the solar neighborhood (Helmi et al. 1999; Smith et al. 2009; Helmi et al. 2017).

Although there are multiple avenues for identifying accreted stars in a halo, isolating an in situ component has been more challenging. Early observations of globular clusters and individual stars indicated that the inner halo is more metalrich, has a metallicity gradient, and is slightly prograde, whereas objects in the outer halo are relatively metal-poor, and retrograde with respect to the Galactic disk (e.g., Searle \& Zinn 1978). This has been interpreted as evidence that the inner halo is formed in situ, whereas the outer halo is accreted. With the advent of wide-field sky surveys, these findings of a dual stellar halo have been confirmed using much larger samples of halo stars (Carollo et al. 2007, 2010; Beers et al. 2012). Adding further evidence for the presence of an in situ component, Schlaufman et al. (2012) ruled out accretion as the main origin of stars in the inner halo, due to their lack of spatial coherence with metallicity. Most recently, Deason et al. (2017) and Kafle et al. (2017) measured a small rotation signal among the old halo stars, which is consistent with the halo having a minor in situ component.

Even though there is abundant evidence that both in situ and accreted stars are present in the Milky Way halo, their contributions have not yet been properly accounted for. A straightforward way to distinguish between these two origin scenarios would be to directly compare halo stars in the Milky Way to a simulated halo, where the origin of every star is known. This comparison is most easily performed by matching stars via their orbital properties, but precise observations of halo stars that would allow such a match have so far been limited to a distance of a few hundred pc from the Sun-a volume poorly resolved in hydrodynamical simulations of large galaxies, such as the Milky Way. Fortunately, major improvements have recently occurred on both the observational and theoretical fronts. The Gaia mission (Perryman et al. 2001) has increased the available observed volume by an order of magnitude. Furthermore, Gaia measurements are much more precise than previously available data, whose role in establishing the presence of a dual halo drew some criticism (Schönrich et al. 2011, 2014). On the theory side, Wetzel et al. (2016) recently presented the Latte high-resolution cosmological zoom-in simulation of a galaxy with mass comparable to the Milky Way. We leverage the joint powers of the new Gaia data set and the Latte simulation to reveal the origin of the stellar halo in the solar neighborhood.

This paper is organized as follows: we start by introducing our observational data that combine the first year of Gaia astrometry with ground-based radial velocity measurements in Section 2. Once we've compiled a data set that has 6D information for stars in the solar neighborhood, we split the sample into a disk and a halo component in Section 3, and analyze their chemical abundances and orbital properties. We interpret these observations in terms of a simple toy model, as well as using the Latte cosmological hydrodynamic simulation of a Milky Way-like galaxy in Section 4, where we also propose a formation scenario for kinematically selected halo stars close to the Sun. Section 5 discusses broader implications of our findings, which are summarized in Section 6.

\section{Data}

Studying orbital properties in a sample of stars requires knowledge of their positions in 6D phase space. Currently, Gaia Data Release 1 (DR1) provides the largest and most precise 5D data set for stars in the solar neighborhood, which we describe in Section 2.1. We complement this data with radial velocities from ground-based spectroscopic surveys whose targets overlap with Gaia (Section 2.2). Finally, we describe our sample selection in Section 2.3.

\subsection{Gaia}

Gaia is a space-based mission that will map our Galaxy over the next several years (Perryman et al. 2001). The first data from the mission were released in 2016 September, and contain not only positions of all Gaia sources $(G<20)$, but also positions, parallaxes, and proper motions for $\sim 2$ million of the brightest stars in the sky (Gaia Collaboration et al. 2016a, 2016b). Obtaining the 5D measurements after just a year of Gaia's operation was made possible by referencing the positions measured with Hipparcos (Michalik et al. 2015). The faintest stars observed by Hipparcos (ESA 1997; van Leeuwen 2007) and released as a part of the Tycho II catalog have $V \sim 12(\mathrm{H} \varnothing \mathrm{g}$ et al. 2000), which limits the size of the 5D sample in Gaia DR1 to $\approx 2$ million stars. A joint solution, known as the Tycho-Gaia Astrometric Solution (TGAS, Lindegren et al. 2016), is comparable in attained proper motions and parallaxes to the Hipparcos precision (typical uncertainty in positions and

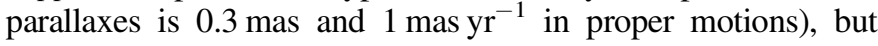
already on a sample that is more than an order of magnitude larger, making TGAS an unrivaled data set for precision exploration of the Galactic phase space.

\subsection{Spectroscopic Surveys}

Gaia is measuring radial velocities for $\sim 150,000$ stars brighter than $G<16$ (Gaia Collaboration et al. 2016b), but the first spectroscopic data will become available only in the second data release. Thus, we completed the phase-space information of TGAS sources by using radial velocities from ground-based spectroscopic surveys. We used two distinct spectroscopic data sets from the Radial Velocity Experiment (RAVE) and Apache Point Observatory Galactic Evolution Experiment (APOGEE) projects, and provide an overview below.

RAVE (Steinmetz et al. 2006) is a spectroscopic survey of the southern sky, with a magnitude range $9<I<12$ wellmatched to TGAS. The latest data release, RAVE DR5 (Kunder et al. 2017), contains $\sim 450,000$ unique radial velocity measurements. Because RAVE avoided targeting regions of low galactic latitude, the actual overlap with TGAS is $\sim 250,000$ stars - the largest of any spectroscopic survey. The survey was performed at the UK Schmidt telescope with the 6dF multi-object spectrograph (Watson et al. 2000), in the wavelength range $8410-8795 \AA$ at a medium resolution of $R \sim 7500$, so the typical velocity uncertainty is $\sim 2 \mathrm{~km} \mathrm{~s}^{-1}$. Abundances of up to seven chemical elements are available for a subset of high signal-to-noise spectra. Casey et al. (2017) reanalyzed the RAVE DR5 spectra in a data-driven fashion with The Cannon (Ness et al. 2015), providing de-noised measurements of stellar parameters and chemical abundances in the RAVE-on catalog. In particular, the typical uncertainty in RAVE-on abundances is 0.07 dex, which is at least $0.1 \mathrm{dex}$ 

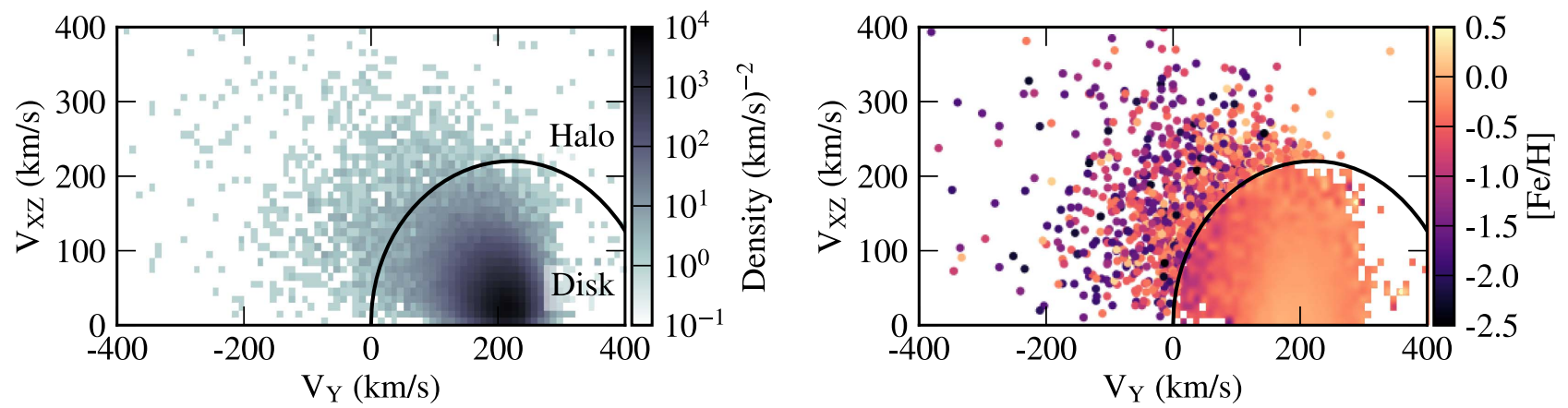

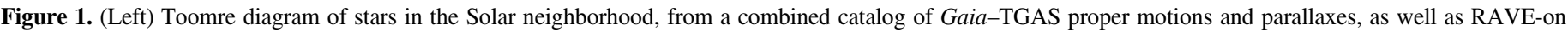

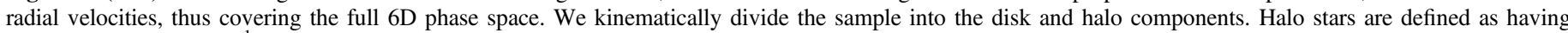

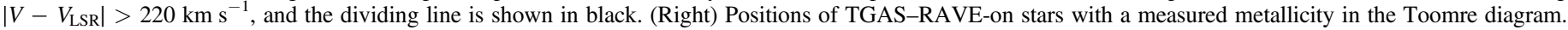

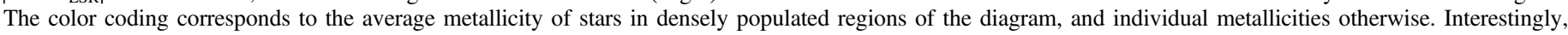
many halo stars are metal-rich, with $[\mathrm{Fe} / \mathrm{H}]>-1$.

better than the precision achieved using the standard spectroscopic pipeline. Therefore, we opted to use RAVE-on chemical abundances, focusing on metallicities, $[\mathrm{Fe} / \mathrm{H}]$, and $\alpha$-element abundances.

The Apache Point Observatory Galactic Evolution Experiment (APOGEE) is one of the programs in the Sloan Digital Sky Survey III (Alam et al. 2015; Majewski et al. 2015), which acquired $\sim 500,000$ infrared spectra for $\sim 150,000$ stars brighter than $H \sim 12.2$ (Holtzman et al. 2015). To capitalize on the infrared wavelength coverage, APOGEE mainly targeted the disk plane, but several high-latitude fields are included as well (Zasowski et al. 2013). Its higher resolution, $R \sim 22,500$, provides more precise abundances for a larger number of elements (e.g., Ness et al. 2015). APOGEE targets are preferentially fainter than stars targeted by RAVE, so its overlap with TGAS is limited to a few thousand stars. APOGEE and RAVE have different footprints, targeting strategies, and observed wavelength windows. Thus, despite the smaller sample size, we found APOGEE to be a useful data set for validating conclusions that were reached by analyzing the larger RAVE sample.

\subsection{Sample Selection}

After matching Gaia-TGAS to the spectroscopic surveys, we increase the quality of the sample by excluding stars with large observational uncertainties. However, the overlap between TGAS and spectroscopic surveys is limited, and the number density of halo stars in the solar neighborhood is low. To ensure that we have a sizable halo sample, we chose to use very generous cuts on observational uncertainties and propagate them when interpreting our results, rather than restricting our sample size by more stringent cuts. In particular, we included stars with radial velocity uncertainties smaller than $20 \mathrm{~km} \mathrm{~s}^{-1}$, and relative errors in proper motions and parallaxes smaller than 1. In addition, we removed all stars with a negative parallax, to simplify the conversion to their distance. These criteria select 159,352 stars for the TGAS-RAVE-on data set, and 14,658 stars for the TGAS-APOGEE sample.

The spatial distribution of stars in our sample is entirely determined by the joint selection function of TGAS and the spectroscopic surveys, as we performed no additional spatial selection. Gaia is an all-sky survey, but the data is still being acquired, so completeness of the TGAS catalog varies across the sky. Ground-based spectroscopic surveys have geographically restricted target lists, in addition to the adopted targeting strategy. This results in a spatially non-uniform sample whose distribution of distances we provide in Appendix A. On the other hand, Wojno et al. (2016) have shown that the RAVE survey is both chemically and kinematically unbiased. Thus, focusing on kinematic properties of the sample will result in robust conclusions.

\section{Properties of the Local Halo Stars}

In this section, we analyze the properties of halo stars in a sample of bright stars, $V \lesssim 12$, within $3 \mathrm{kpc}$ of the Sun, that have positions, proper motions, and parallaxes in the TGAS catalog, and radial velocities from either RAVE or APOGEE. This sample, though spatially incomplete due to survey selection functions, is kinematically unbiased. We define a kinematically selected halo in Section 3.1, and present its chemical and orbital properties in Sections 3.2 and 3.3, respectively.

\subsection{Defining a Local Sample of Halo Stars}

Access to the full $6 \mathrm{D}$ phase space information allows us to calculate Galactocentric velocities for all of the stars in the sample. We summarize the kinematic properties of the sample with a Toomre diagram (Figure 1), where the Galactocentric $Y$ component on the velocity vector, $V_{Y}$ (which points in the direction of the disk rotation), is on the $x$ axis, whereas the perpendicular Toomre component, $\sqrt{V_{X}^{2}+V_{Z}^{2}}$, is on the $y$ axis. This space has been widely used to identify major components of our Galaxy, such as the thin and thick disks, and the halo (e.g., Venn et al. 2004). Disk stars dominate a large overdensity at $V_{Y} \approx 220 \mathrm{~km} \mathrm{~s}^{-1}$, which corresponds to the circular velocity of the Local Standard of Rest (LSR, $V_{\mathrm{LSR}}$ ). The density of stars (left panel of Figure 1) decreases smoothly for velocities that are progressively more different from $V_{\mathrm{LSR}}$, extending all the way to retrograde orbits $\left(V_{Y}<0\right)$.

We distinguish between the disk and halo following Nissen $\&$ Schuster (2010): halo stars are identified with a velocity cut $\left|V-V_{\mathrm{LSR}}\right|>220 \mathrm{~km} \mathrm{~s}^{-1}$, where $V_{\mathrm{LSR}}=(0,220,0) \mathrm{km} \mathrm{s}^{-1}$ in the Galactocentric Cartesian coordinates. The dividing line between the components is marked with a black line in Figure 1, and both components are labeled in the left panel. The halo definition employed here is more conservative than similar cuts adopted by previous studies; e.g., Nissen \& Schuster 
(2010) defined halo as stars with velocities that satisfy $\left|V-V_{\mathrm{LSR}}\right|>180 \mathrm{~km} \mathrm{~s}^{-1}$. For example, Schönrich \& Binney (2009a) have shown that the velocity distribution of a Galactic thick disk can be asymmetric, in which case the region $180<\left|V-V_{\mathrm{LSR}}\right|<220 \mathrm{~km} \mathrm{~s}^{-1}$ could still contain many thick disk stars. A higher velocity cut ensures that the contamination of our halo sample with thick disk stars is minimized. In total, we identified 1376 halo and 157,976 disk stars, with the halo constituting $\sim 1 \%$ of our sample. This is in line with the expectations from number count studies in large-scale surveys (e.g., Jurić et al. 2008), although we do not expect an exact match, as TGAS is not volume complete.

\subsection{Chemical Composition}

In this section, we study the chemical composition of the solar neighborhood stars observed by both Gaia-TGAS and RAVE. The signal-to-noise ratio of 142,086 RAVE spectra was high enough to allow a measurement of metallicity $[\mathrm{Fe} / \mathrm{H}]$. Alpha-element abundances, $[\alpha / \mathrm{Fe}]$, were obtained for a subset of 56,259 stars. All of the RAVE elemental abundances used in this work were measured by Casey et al. (2017). Right panel of Figure 1 shows the average metallicity in densely populated velocity bins of the Toomre diagram, whereas the points in the lower-density regions are individually colored-coded by $[\mathrm{Fe} / \mathrm{H}]$. As expected, the halo is poorer in metallicity than the disk (e.g., Gilmore et al. 1989; Ivezić et al. 2008). Within the disk itself, there is a smooth decrease in metallicity further from the $V_{\mathrm{LSR}}$, starting from $[\mathrm{Fe} / \mathrm{H}] \sim 0$ in the thin disk region, $\left(V_{Y}, V_{X Z}\right)=(220,0) \mathrm{km} \mathrm{s}^{-1}$, to $[\mathrm{Fe} / \mathrm{H}] \sim-0.5$ in the thick disk region, $\left(V_{Y}, V_{X Z}\right)=(100,100) \mathrm{km} \mathrm{s}^{-1}$. Surprisingly, however, there are many stars with thick disk-like metallicities found in the halo region of the Toomre diagram, and some of them are on very retrograde orbits.

Figure 2 (top) shows the metallicity distribution for the two kinematic components identified above: the disk in red and the halo in blue. The disk is richer in metallicity than the halo, and peaks at the approximately solar metallicity, $[\mathrm{Fe} / \mathrm{H}]=0$. The halo is relatively metal-poor, and exhibits a peak at $[\mathrm{Fe} / \mathrm{H}] \sim-1.6$, typical of the inner halo (e.g., Allende Prieto et al. 2006). However, the metallicity distribution of the halo has an additional peak at the metal-rich end, centered on $[\mathrm{Fe} / \mathrm{H}] \sim-0.5$ and extending out to the super-solar values.

To corroborate the existence of metal-rich stars on halo orbits, we also show the metallicity distribution function for TGAS stars observed with APOGEE at the bottom of Figure 2. We used APOGEE elemental abundances provided as a part of the SDSS IV Data Release 13 (SDSS Collaboration et al. 2016). The disk-halo decomposition for the APOGEE sample was performed in a manner identical to that of RAVE-on. The metallicity distributions between the two surveys are similar: the disk stars are metal-rich, whereas the halo has a wide distribution ranging from $[\mathrm{Fe} / \mathrm{H}] \sim-2.5$ to $[\mathrm{Fe} / \mathrm{H}] \sim 0$. A bimodality is present in the metallicity distribution of APOGEE halo stars, although it is less prominent than in the RAVE-on sample due to the smaller sample size. The apparent bimodality in the metallicity distribution of RAVE-on halo stars is slightly more metal-poor, $[\mathrm{Fe} / \mathrm{H}] \approx-1.1$, than observed in the APOGEE sample, $[\mathrm{Fe} / \mathrm{H}] \approx-0.8$. In what follows, we compromise between these two values and split the halo sample at $[\mathrm{Fe} / \mathrm{H}]=-1$, into a metal-rich $([\mathrm{Fe} / \mathrm{H}]>-1)$ and a metal-poor component $([\mathrm{Fe} / \mathrm{H}] \leqslant-1)$. With 598 out of 941 RAVE-on halo stars $(64 \%)$ and 22 out of 50 APOGEE halo
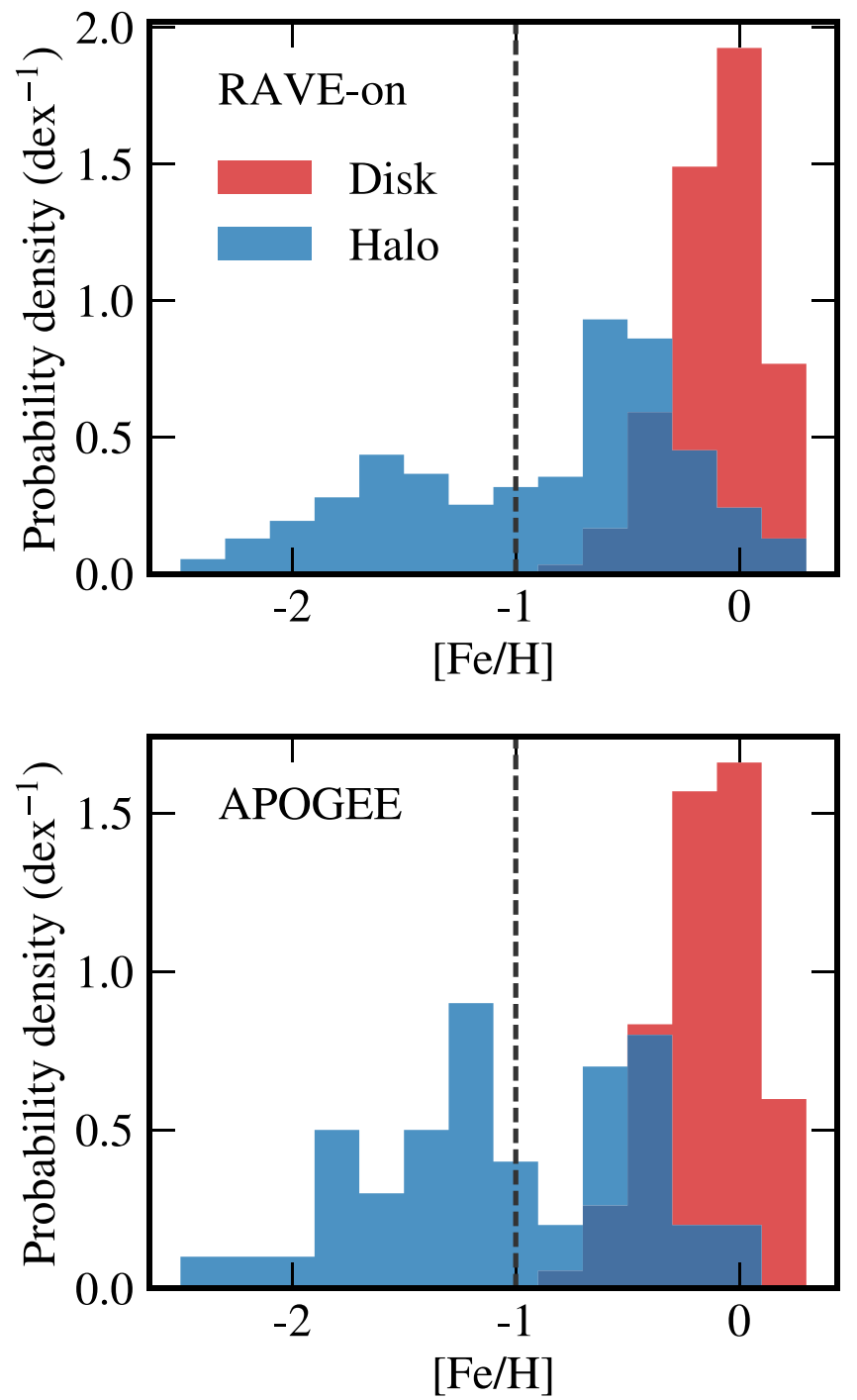

Figure 2. Metallicity distribution function of the solar neighborhood in the TGAS and RAVE-on (top), and TGAS and APOGEE catalogs (bottom). Kinematically selected disk stars are shown in red, whereas the halo distribution is plotted in blue. In both samples, there is a population of metal-rich halo stars, with $\approx 50 \%$ of stars having $[\mathrm{Fe} / \mathrm{H}]>-1$ (marked with a vertical dashed line).

stars $(44 \%)$ having $[\mathrm{Fe} / \mathrm{H}]>-1$, approximately half of the halo sample is metal-rich.

Chemical abundances have been used to discern different components of the Galaxy (e.g., Gilmore et al. 1989). The abundance space of $[\alpha / \mathrm{Fe}]$ versus $[\mathrm{Fe} / \mathrm{H}]$ is particularly useful in tracing the origin of individual stars (e.g., Lee et al. 2015). Figure 3 shows this space for RAVE-on spectra on the top, and APOGEE on the bottom. The disk distribution is shown as a red density map, while the less numerous halo stars are shown individually in blue. Similarly to the overall metallicity distribution function, RAVE-on and APOGEE surveys are in qualitative agreement in terms of the more detailed chemical abundance patterns. At low metallicities, the halo is $\alpha$ enhanced, but its $[\alpha / \mathrm{Fe}]$ declines at high metallicities, following the disk abundance pattern, both in terms of the mean $[\alpha / \mathrm{Fe}]$ and its range at a fixed $[\mathrm{Fe} / \mathrm{H}]$. In particular, thick-disk stars have higher $\alpha$-element abundances at a given metallicity (e.g., Nidever et al. 2014) and follow a separate 

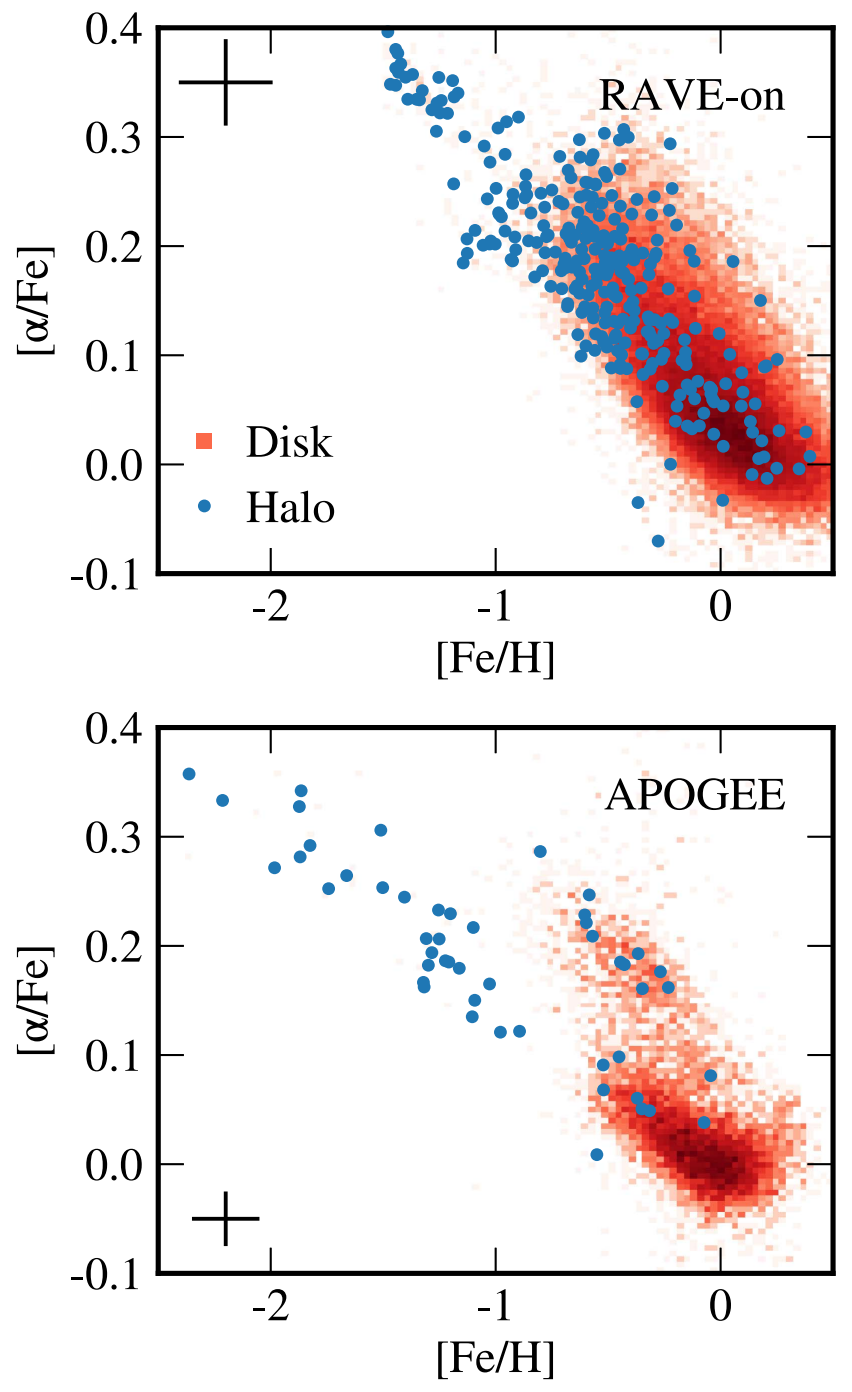

Figure 3. Chemical abundance pattern, $[\alpha / \mathrm{Fe}]$ vs. $[\mathrm{Fe} / \mathrm{H}]$, for the TGASRAVE-on sample (top) and TGAS-APOGEE (bottom). The pattern for disk stars is shown as a red-colored Hess diagram (logarithmically stretched), and halo stars are shown individually as blue points. In both surveys, the metalpoor halo is $\alpha$-enhanced, while the metal-rich halo follows the abundance pattern of the disk.

sequence visible in the more precise APOGEE data $([\mathrm{Fe} / \mathrm{H}] \sim-0.2,[\alpha / \mathrm{Fe}] \sim 0.2)$, whereas the thin disk is generally more metal-rich and $\alpha$-poor. Metal-rich halo stars in both samples span this range of high- and low- $\alpha$ abundances. At lower metallicities, Nissen \& Schuster (2010) reported that halo stars follow two separate $[\alpha / \mathrm{Fe}]$ sequences, with the high$\alpha$ stars being on predominantly prograde orbits, whereas the low- $\alpha$ stars are mostly retrograde. We neither resolve the two sequences nor see any correlation between the orbital properties and $[\alpha / \mathrm{Fe}]$ at a fixed $[\mathrm{Fe} / \mathrm{H}]$. However, the RAVE-on abundances are fairly uncertain (typical uncertainty is $\sim 0.07$ dex, marked by a black cross on the top left in Figure 3), so the sequences seen in the higher-resolution data from Nissen \& Schuster (2010) would not be resolved in this data set. In the APOGEE sample, where the typical uncertainty is smaller, $\sim 0.03$ dex, the number of halo stars is too small to unambiguously identify multiple $[\alpha / \mathrm{Fe}]$ sequences.

The evidence presented so far shows that the stellar halo in the solar neighborhood has distinct metal-poor and metal-rich

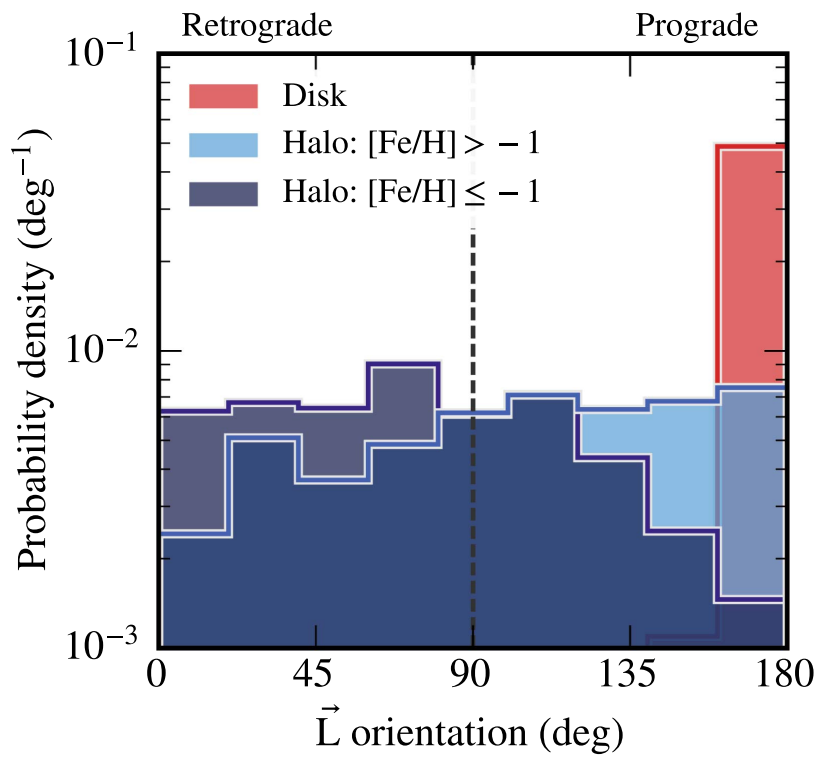

Figure 4. Orientation of angular momenta, based on RAVE-on kinematics, with respect to the Galactocentric $Z$ axis for different Galactic components: the disk is in red, metal-poor halo in dark blue, and metal-rich halo in light blue. The angular momenta of disk stars are aligned with the $Z$ axis, whereas those of halo stars are more uniformly distributed. There is an excess of metal-rich halo stars on prograde orbits, $\theta_{L}>90^{\circ}$, compared to the metal-poor halo orbital orientations.

components. Given that the metal-rich component follows the abundance pattern of the disk, we discuss possible contamination by the thick disk in Appendix B, and rule out the possibility that a sizable fraction of the metal-rich halo is attributable to the canonical thick disk. In the next section, we proceed to characterize the orbital properties of the two halo components.

\subsection{Angular Momenta}

Stellar orbits can be classified in terms of their integrals of motion. However, calculating these requires the knowledge of the underlying gravitational potential (Binney \& Tremaine 2008). Furthermore, in a realistic Galactic environment, some stars are on chaotic orbits (e.g., Price-Whelan et al. 2016), where the integrals of motion do not exist. On the other hand, any star with a measured position in a $6 \mathrm{D}$ phase space has a well-defined angular momentum. In this section, we use angular momenta as empirical diagnostics of stellar orbits, and focus particularly on the orientation of the angular momentum vector with respect to the Galactocentric $Z$ axis, quantified by the angle

$$
\theta_{L} \equiv \arctan \left(L_{Z} /|\boldsymbol{L}|\right)
$$

where $L_{Z}$ is the $Z$ component of the angular momentum, and $|\boldsymbol{L}|$ its magnitude. Here, $L_{Z}$, and hence $\theta_{L}$, are conserved quantities in static, axisymmetric potentials, such as that of the Milky Way disk. This has already been utilized to identify coherent structures in the phase space of local halo stars (e.g., Helmi et al. 1999; Smith et al. 2009). In the adopted coordinate system, the disk orientation angle is $\theta_{L}=180^{\circ}$, such that prograde orbits are those with $\theta_{L}>90^{\circ}$, and retrograde have $\theta_{L}<90^{\circ}$. Figure 4 shows the distribution of angular 
momentum orientations $\theta_{L}$ for the Galactic components identified in the RAVE sample.

As expected, most of the disk stars are indeed on orbits in the disk plane with $V_{Z} \approx 0$, and have $\theta_{L} \approx 180^{\circ}$ (red histogram in Figure 4). Angular momenta of both halo components span the entire range of $0^{\circ}<\theta_{L}<180^{\circ}$. However, in detail, their distributions are significantly different from each other. The metal-poor halo has an almost flat distribution as a function of $\theta_{L}$, with a slight depression at very prograde angles (dark blue histogram in Figure 4). The metal-rich halo is predominantly prograde $\left(63 \%\right.$ of the stars have $\left.\theta_{L}>90^{\circ}\right)$, but also has a long tail to retrograde orbits (light blue histogram in Figure 4). In the next section, we explore the origin of these distributions in terms of a toy model for the kinematic distribution of the Galaxy, as well as in comparison to a hydrodynamical simulation of a Milky Way-like galaxy.

\section{Origin of Halo Stars in the Solar Neighborhood}

In the previous section, we presented a metal-rich component of the stellar halo in the solar neighborhood that moves preferentially in prograde orbits with respect to the Galactic disk. In this section, we test whether the observed orientations of these angular momenta can be understood in the context of a simple toy model (Section 4.1), as well as in comparison to a solar-like neighborhood in a cosmological hydrodynamical simulation (Section 4.2). Finally, we study the formation paths of the simulated stellar halo, to provide a possible origin for this metal-rich halo component (Section 4.3).

\subsection{Toy Model}

To construct a toy model for our TGAS-RAVE-on sample, we assign stars to one of the three main components of the Milky Way galaxy: a thin disk, thick disk, or halo (e.g., BlandHawthorn \& Gerhard 2016). The model is defined by the number of stars in each component, and their spatial and kinematic properties. To set the number of halo stars in the toy model, we assumed that the halo is isotropic. In that case, an equal number of halo stars are on prograde and retrograde orbits. Because we expect no contamination from the disk on retrograde orbits, we set the total size of the halo in the toy model to be twice the number of retrograde stars in our Milky Way sample. For the remaining disk stars, we vary the ratio of thin to thick disk stars to best match the distribution of prograde orbits.

Once the number of stars in each component is determined, we proceed to assign them their phase space coordinates. Our sample is spatially confined to within only several kpc from the Sun (see Appendix A), so we see no differences in spatial distributions of the kinematically defined components from Section 2.3. This allows us to take the spatial distribution of stars in our sample, and randomly designate them a component in the toy model, thus ensuring that the spatial selection function of both TGAS and RAVE-on is properly reproduced in the model. For each star in the model, we draw a 3D velocity from its component's velocity ellipsoid measured by Bensby et al. (2003) on a smaller sample of local stars with Hipparcos parallaxes and proper motions, which accounts for the asymmetric drift. With positions and velocities in place, we calculate the angular momenta and their orientation angles with respect to the $Z$ axis, $\theta_{L}$, for all stars in the toy model.
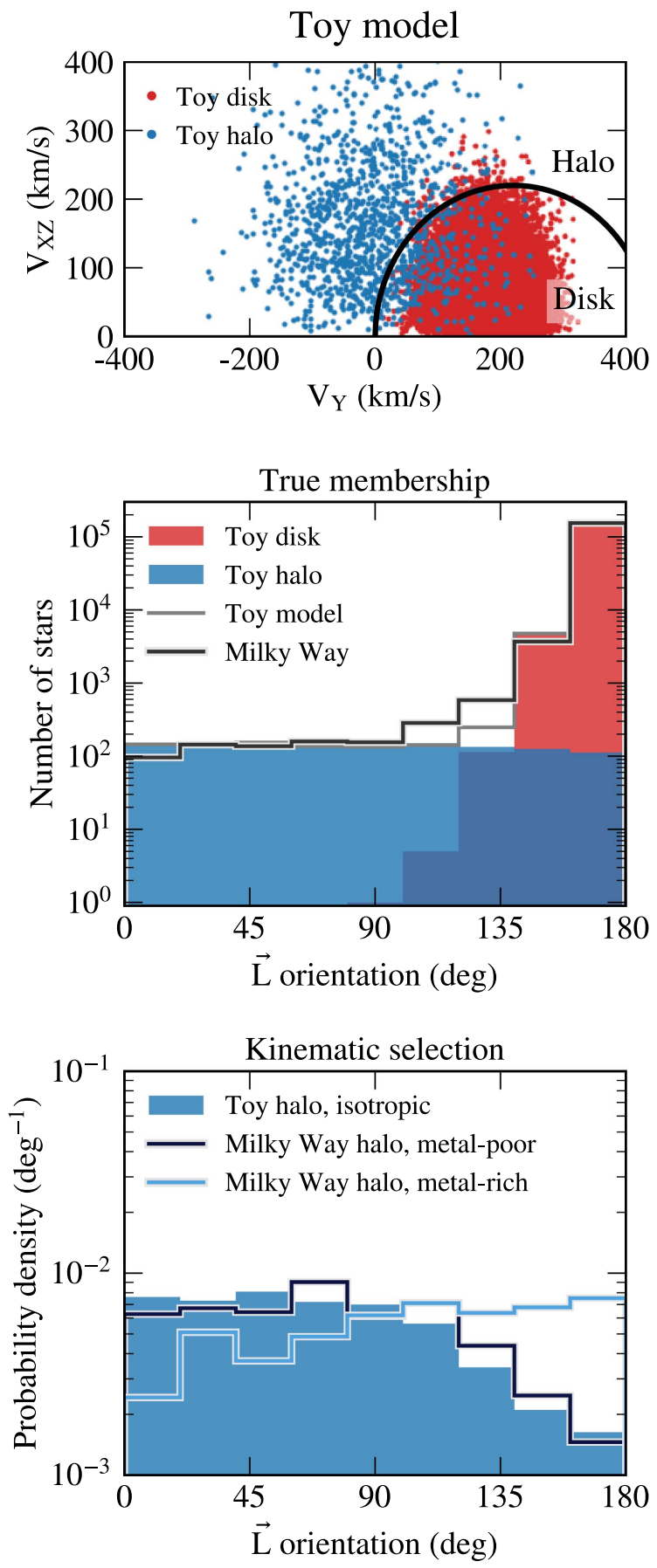

Figure 5. Toy model for the phase space of the solar neighborhood. The model consists of a halo (blue) and a disk component (red), with their positions drawn directly from the TGAS-RAVE-on sample, and kinematics from the velocity ellipsoids of Bensby et al. (2003). Top panel shows the model in the Toomre diagram. The black line is the employed demarcation between the halo and the disk, which does fairly good job of separating the two in the toy model, as well. The middle panel shows the orientation of angular momenta in the model, with each component shown as a shaded histogram, and a model total with a black line. Toy model angular momenta successfully reproduce the angular momentum orientations observed in the Milky Way (gray dashed line). Kinematically selecting the halo in the model (bottom panel, shaded histogram) produces a distribution in excellent agreement with the distribution of metalpoor halo stars in the Milky Way (dark blue line). The metal-rich halo in the Milky Way (light blue line) is inconsistent with being a part of an isotropic halo studied in this toy model.

The properties of our toy model are summarized in Figure 5. The top panel shows the components of the model in the Toomre diagram, with the disk stars in red, halo stars in blue, 
and the black line delineating our kinematic boundary between the halo and the disk. The velocity ellipsoids of these components overlap, so our kinematic definition of a halo produces a sample that is likely neither pure nor complete. This is illustrated in the toy model by several thick-disk stars that enter the halo selection box at $\left(V_{Y}, V_{X Z}\right) \simeq(100,200) \mathrm{km} \mathrm{s}^{-1}$, as well as halo stars on prograde orbits, which fall outside of the halo selection. As no simple kinematic cut will completely separate the different components, we opt to emphasize the purity of our halo sample. Based on the toy model, we estimate that the fraction of interloping disk stars in a kinematically defined halo is only $\sim 10 \%$, but this in turn makes the halo sample less complete at $75 \%$. For a comparison, Nissen \& Schuster (2010) define a halo that is $\sim 90 \%$ complete, but only $\sim 55 \%$ pure.

The middle panel of Figure 5 shows the orientation of angular momenta, $\theta_{L}$, for the components of the toy model, with halo in blue and disk in red. As expected, the majority of disk stars are moving in the disk plane, and the distribution is sharply peaked at $\theta_{L}=180^{\circ}$. The nearly isotropic halo of the Bensby et al. (2003) model has a flat distribution in $\theta_{L}$. The sum of the two components is represented with a thick black line, which compares favorably to the distribution of $\theta_{L}$ observed in the Milky Way, and shown in dashed gray. The agreement between the toy model and the Milky Way is particularly good at very prograde or retrograde orbits. The abundance of stars on only slightly prograde orbits $\left(\theta_{L} \approx 100^{\circ}\right)$ is somewhat underestimated in the toy model, indicating that the transition between a disk and a halo component in the Milky Way is more gradual than what can be reproduced by a simple model that features only two disks and an isotropic halo.

The bottom panel of Figure 5 compares the modeled halo to the observed one. For a fair comparison, in this panel we only consider model stars that would satisfy our kinematic halo selection, which are a combination of some disk and the majority of halo stars, as discussed above. The distribution of $\theta_{L}$ for these kinematicallyselected halo stars from a toy model is shown as a shaded histogram. Although the intrinsic distribution of an isotropic halo is flat (middle panel), kinematic selection introduces a suppression at the most prograde orbits (bottom panel). Kinematic selection excludes halo stars with $\left|V-V_{\mathrm{LSR}}\right| \leqslant 220 \mathrm{~km} \mathrm{~s}^{-1}$, all of which are on prograde orbits, which is manifested as a depression at $\theta_{L} \gtrsim 90^{\circ}$. The magnitude of this depression exactly matches the distribution of metal-poor halo stars in the Milky Way, overplotted with a dark blue line in the bottom panel of Figure 5. This suggests that the metal-poor halo in the solar neighborhood is intrinsically isotropic, and that at least some stars more metal-poor than $[\mathrm{Fe} / \mathrm{H}] \leqslant-1$, which are kinematically consistent with the disk, are in fact a part of the metal-poor halo. The distribution of metalrich halo stars in the Milky Way, shown as light blue line in the bottom panel of Figure 5, shows the opposite behavior of an excess at prograde orbits, and is significantly different from the toy model prediction for an isotropic halo.

A simple toy model successfully explains the bulk properties of our sample: most of the stars are in a rotating disk, with a minority in an isotropic halo, which maps well to the metalpoor halo stars identified in our TGAS-RAVE-on sample. This toy model also highlights that the metal-rich halo stars are inconsistent with either of these components. Next, we analyze the origin of such a population using a hydrodynamical simulation.

\subsection{The Latte Simulation}

The Latte simulation, first presented in Wetzel et al. (2016), comes from the Feedback In Realistic Environments (FIRE) ${ }^{8}$ project (Hopkins et al. 2014). Latte is fully cosmological, with baryonic mass resolution of $7000 M_{\odot}$, and spatial softening/ smoothing of $1 \mathrm{pc}$ for gas and $4 \mathrm{pc}$ for stars. The simulation uses the standard FIRE-2 implementation of gas cooling, star formation, stellar feedback, and metal enrichment described in Hopkins et al. (2017), including the numerical terms for turbulent diffusion of metals in gas described therein ${ }^{9}$, and the GIZMO code (Hopkins 2015). FIRE simulations have been used to study galaxies ranging from ultra-faint dwarf galaxies (Wheeler et al. 2015) to small groups of galaxies (Feldmann et al. 2016). FIRE simulations successfully reproduce such features as: observed internal properties of dwarf galaxies (Chan et al. 2015; El-Badry et al. 2016); thin/thick disk structure, and both radial and vertical disk metallicity gradients in a Milky Way-like galaxy (Ma et al. 2016b); star-formation histories of dwarf and massive galaxies (Hopkins et al. 2014; Sparre et al. 2015); global galaxy scaling relations (Hopkins et al. 2014; Feldmann et al. 2016; Ma et al. 2016a); and satellite populations around Milky Way-mass galaxies (Wetzel et al. 2016).

To construct a sample of star particles in Latte analogous to the TGAS-RAVE-on sample, we first align the simulation coordinate system with the disk, and then select stars in a $3 \mathrm{kpc}$ sphere located at a distance of $8.3 \mathrm{kpc}$ from the center of the galaxy. We classify star particles as either disk or halo, using a kinematic cut in the Toomre diagram similar to the one employed for stars in the Milky Way (Section 3.1). Because the circular velocity in Latte is slightly different from the Galactic value, the definition of the local standard of rest is also different, but we keep the same conservative measure for the dispersion of $220 \mathrm{~km} \mathrm{~s}^{-1}$ in the disk of Latte. The Toomre diagram for the sample in Latte is shown in the top panel of Figure 6. Qualitatively, it is similar to that of the Milky Way (Figure 1), with most of the stars rotating in the disk at $\sim 235 \mathrm{~km} \mathrm{~s}^{-1}$, and the density of stars smoothly decreasing away from the local standard of rest. Quantitatively, the halo fraction in Latte is an order of magnitude higher at $10 \%$. Its kinematic space is also more structured, compared to the Milky Way. This is partly because the Latte sample does not suffer from selection effects, so it effectively extends to larger distances, where the halo constitutes a larger mass fraction. Any kinematic structures are hence better sampled and more readily observable in Latte. Additionally, at least some of the structure present in the Milky Way sample is smoothed by the observational uncertainties (see, e.g., Sanderson et al. 2015), which are not present in Latte.

Stars in the Toomre diagram of Figure 6 are color-coded by metallicity and show trends similar to those observed in the Milky Way. For a more quantitative analysis, we show metallicity distribution of Latte's disk and halo components in the central panel of Figure 6. To facilitate comparison, we

\footnotetext{
8 The FIRE project website is http://fire.northwestern.edu.

9 The original simulation presented in Wetzel et al. (2016) did not include terms for turbulent metal diffusion. Here, we analyze a re-simulation that includes those terms (with all other parameters unchanged), which creates a more realistic metallicity distribution function in both the host galaxy (A. Wetzel et al. 2017, in preparation) and its satellites (I. Escala et al. 2017, in preparation). As explored in Su et al. (2016) and Hopkins et al. (2017), the inclusion of turbulent metal diffusion has no systematic effect in any gross galaxy properties (including the average metallicity), as we also checked for all analyses in this paper.
} 


\section{Latte simulation}

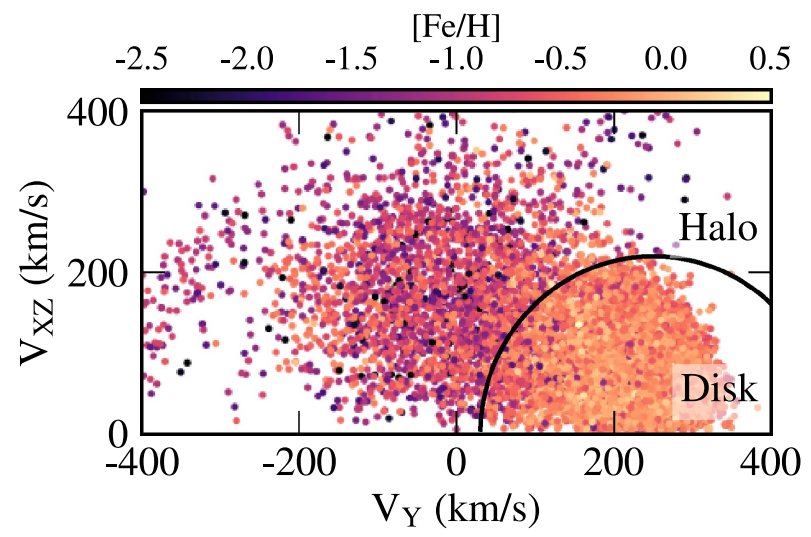

Kinematic selection
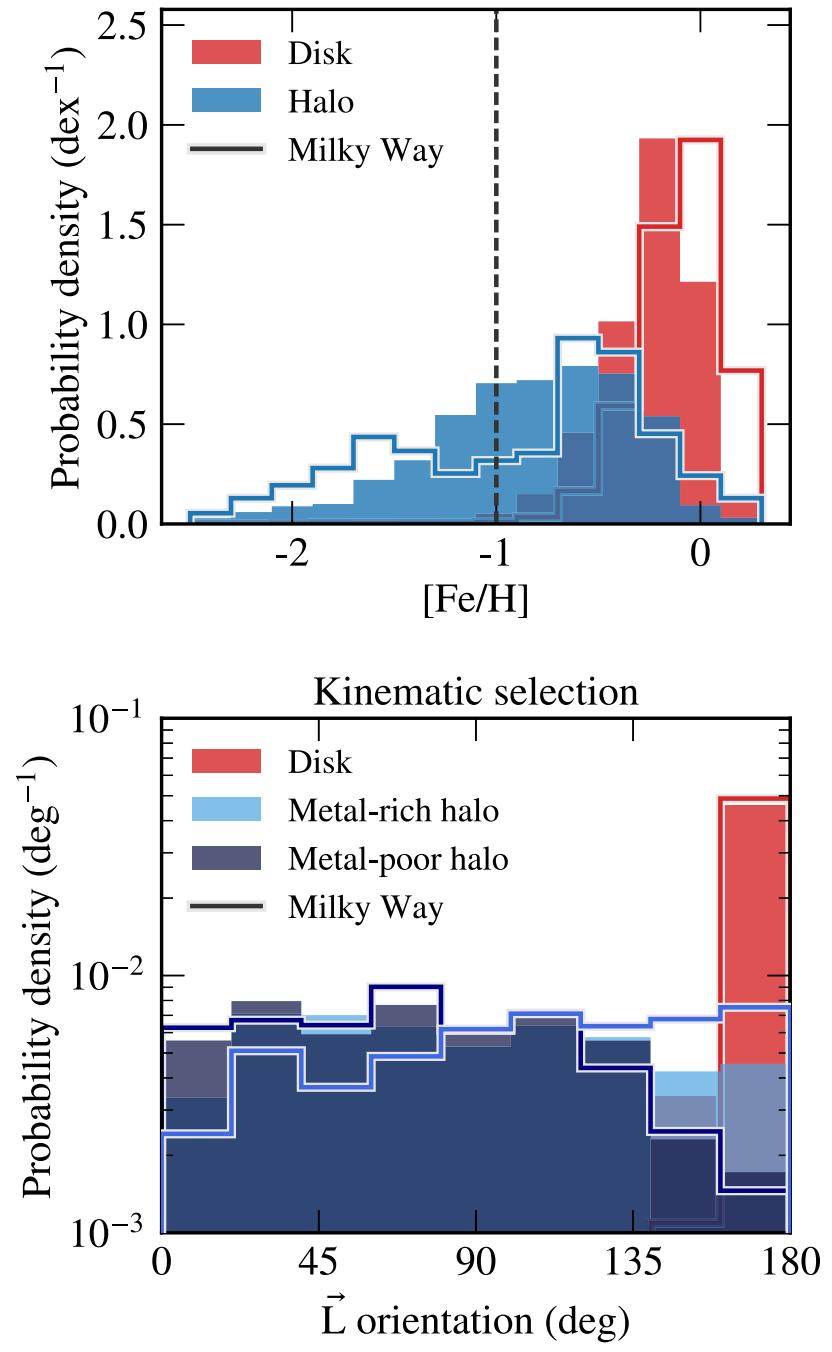

Figure 6. Stars from the Latte cosmological zoom-in baryonic simulation of a Milky Way-mass galaxy, from the FIRE project, observed identically to stars in the solar neighborhood. Top panel shows positions of stars in the Toomre diagram, color-coded by metallicity. We identify disk and halo stars in Latte by using a cut in this diagram (black line), analogous to the cut used for the Milky Way sample. Metallicity distributions of Latte stars are in the middle, with the disk being shaded red, and halo blue. Bottom panel shows the orientations of stars' angular momenta in Latte, with the disk in red, metal-poor halo in dark blue, and metal-rich halo in light blue. Properties of Latte stars are remarkably similar to the distributions of stars in our Milky Way sample (reproduced as empty histograms on the middle and bottom panel, for direct comparison). also include metallicity distributions of the Milky Way components as empty histograms. The Latte halo is poorer in metallicity than its disk, and although there is no bimodality in the halo metallicity, the whole distribution is as wide as observed in the Milky Way-extending from $[\mathrm{Fe} / \mathrm{H}] \lesssim-2$ to $[\mathrm{Fe} / \mathrm{H}] \simeq 0$. This agreement, in addition to $[\alpha / \mathrm{Fe}]$ abundance trends recovered in simulated disks (A.Wetzel et al. 2017, in preparation), demonstrates that the physics included in the FIRE simulations captures the most important ingredients for chemical evolution in galaxies. Following our analysis of the Milky Way sample, we proceed to divide the Latte halo into a metal-rich component with $[\mathrm{Fe} / \mathrm{H}]>-1$, and a metal-poor one with $[\mathrm{Fe} / \mathrm{H}] \leqslant-1$. The ratio of metal-rich to metal-poor halo stars in Latte is not quite the same as in the Milky Way, but this does not seriously impede our goal of qualitatively understanding differences between the two populations.

Finally, we analyze orbital properties of stars in Latte by showing the orientation of their angular momenta with respect to the $Z$ axis, $\theta_{L}$, as solid histograms in the bottom panel of Figure 6. The angular momenta of Latte disk stars are wellaligned with the $z$-axis, with $\theta_{L} \simeq 180^{\circ}$, similar to disk stars in the Milky Way (shown in Figure 6 as an empty histogram). The Latte halo shows a flatter distribution of $\theta_{L}$, but there is still an excess of metal-rich stars on prograde orbits (histogram shaded light blue) with respect to the metal-poor halo stars (dark blue). Overall, Latte stars have similar kinematic, chemical, and orbital properties to stars observed in the Milky Way, suggesting that it provides a reasonable analogue. We next trace Latte stars back to their birth location and suggest a possible scenario for the formation of halo stars in the solar neighborhood.

\subsection{Formation Paths of the Latte Stellar Halo}

We define the formation distance of a star particle in Latte as its physical (not comoving) distance from the center of the primary (Milky Way-like) galaxy at the time that it formed. We inspect this formation distance as a function of the star's current $(z=0)$ age in Figure 7. Stars with disk kinematics (as defined by our Toomre-diagram cut in Figure 6) are shown in red, whereas those identified as halo stars are blue. The suppression of stars that formed at solar distances $\sim 7 \mathrm{Gyr}$ ago coincides with the time of the last significant merger $(z \sim 0.7)$. This event brought significant gas to the center of the galaxy, thus switching the star-forming conditions from those producing mainly stars on halo-like orbits (more than $95 \%$ of halo stars are older than $6 \mathrm{Gyr}$ ) to the orderly production of disk stars (more than $90 \%$ of disk stars were formed in the last $6 \mathrm{Gyr})$. The formation distances between the disk and halo components are equally dichotomous: most of the disk stars were formed close to their present-day distance from the galactic center (5-11 kpc, shaded gray in Figure 7), whereas halo stars originated from the extremes of the central $1 \mathrm{kpc}$ to the virial radius and beyond (for reference, the virial radius $10 \mathrm{Gyr}$ ago was $\approx 70 \mathrm{kpc}$ ). The distribution of formation distances for the metal-rich and metal-poor halo components is shown on the right, with light and dark blue histograms, respectively. Metal-poor halo stars originate from a range of distances, with the median of the distribution at $\sim 45 \mathrm{kpc}$. On the other hand, the median formation distance of the metal-rich halo stars is $\sim 5 \mathrm{kpc}$, closer to the Galactic center than their current position. Overall, only $\sim 20 \%$ of Latte stars on halo orbits were formed within their present-day radial distance range, indicating that radial redistribution is an important 


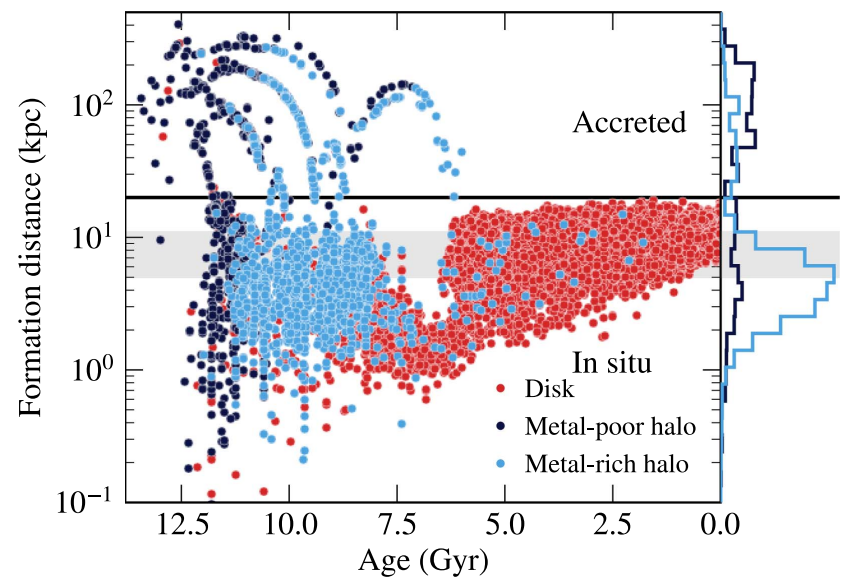

Figure 7. Distance from the host galaxy at the time of formation, as a function of current stellar age (in physical kpc), for star particles currently in the solar neighborhood (gray shaded region) in the Latte simulation. Blue circles are kinematically identified as a halo (with metal-rich stars plotted in light blue, and metal-poor in dark blue), and red circles are kinematically consistent with the disk. The galaxy experienced a significant merger 7 Gyr ago, so most stars formed at that time come from the inner $1 \mathrm{kpc}$. Current disk stars formed in situ (within $20 \mathrm{kpc}$ ) and at later times. The halo formed early, and has both an in situ and an accreted component (stars formed outside of $20 \mathrm{kpc}$ ). The metalpoor halo preferentially formed at larger distances than the metal-rich halo (right histograms).

phenomenon sculpting the inner Galaxy. We discuss the implications of the halo component undergoing radial migration/ heating in Section 5.2.2.

The formation distance of a star particle can also be used as a rudimentary diagnostic of its formation mechanism. From Figure 7, we see that almost none of the disk stars were formed beyond $20 \mathrm{kpc}$ (indicated with a horizontal black line), so we adopt this distance as a delimiter between the in situ and accreted origin for stars in Latte. Most of the accreted stars are classified as halo, and were formed inside dwarf galaxies merging with the host galaxy. The tracks in the space of formation distance and age (Figure 7) delineate the orbits of these dwarf galaxies. All of the satellites are disrupted once they get within the central $20 \mathrm{kpc}$; for most of them, this happens on the first approach. In the process, they bring in gas that fuels in situ star formation. At early times, most of the stars that formed in situ also become a part of the halo, whereas the last significant merger starts the onset of the in situ formation of the disk. Although satellite accretion onto the host galaxy continues after the last significant merger, none of the accreted stars reach the solar circle, so effectively all of the halo stars in the solar neighborhood were formed prior to the last significant merger. This is in line with findings of Zolotov et al. (2009), who showed that late-time accretion predominantly builds outer parts of the stellar halo.

In summary, Latte stars born at late times formed in the disk, whereas old stars in Latte are predominantly part of the halo. One third of the halo in the solar neighborhood was accreted from the infalling satellites, whereas the majority of stars formed in situ in the inner galaxy and migrated to the solar circle. In this simplified depiction for the origin of the stellar halo, we do not account for the unlikely possibility that stars that formed within $20 \mathrm{kpc}$ still could have been bound to a satellite galaxy, and hence accreted, nor do we distinguish between different modes of in situ halo formation-e.g., through a dissipative collapse (Samland \& Gerhard 2003) or with stars being heated from the disk (Purcell et al. 2010). These are not serious short comings because satellites that entered the inner $20 \mathrm{kpc}$ were disrupted soon thereafter, so any newly formed stars would have been only loosely bound to the satellite at the time. Furthermore, given that the median formation distance of all halo stars formed in situ is $\sim 4 \mathrm{kpc}$, we expect that dissipative collapse only marginally contributes to the census of halo stars at the solar circle, and ultimately, we draw conclusions that are insensitive to the particulars of their origin.

In Figure 8, we explore how the fraction of halo stars formed in situ depends on the angular momentum orientation $\left(\theta_{L}\right.$, left) and a combination of angular momentum and metallicity (right). On average, $40 \%$ of metal-poor halo stars have formed in situ (dark blue line in the left panel of Figure 8). Metal-rich halo stars are more likely to have formed in situ (light blue line), and this probability increases to $90 \%$ for stars whose orbits are aligned with the disk. Interestingly, when we investigate the dependence of the in situ fraction simultaneously as a function of metallicity and angular momentum orientation (right panel of Figure 8), we find only a weak dependence on the current angular momentum orientation of a star, but a strong correlation with metallicity. All of the lowestmetallicity stars, with $[\mathrm{Fe} / \mathrm{H}] \lesssim-2.5$, were accreted (yellow), and all of the metal-rich halo stars, with $[\mathrm{Fe} / \mathrm{H}] \gtrsim-0.5$, formed in situ (purple). Given the similarities between the global chemical and orbital properties in Latte and the Milky Way, this result suggests that the metal-rich halo component identified in the TGAS-RAVE-on sample formed in the inner Galaxy, but was driven to the solar circle through subsequent evolution.

Ages of individual stars are another important diagnostic of their origin, so we now explore correlations between metallicity and age for stars in the solar neighborhood, as predicted by the Latte simulation. Figure 9 shows how the metallicity of stars in Latte depends on their age for the disk (red), in situ halo (light blue), and accreted halo (dark blue). Shaded regions correspond to the 16-84th percentiles in the distribution of metallicities for stars of a given age. In general, metallicity increases with time. However, accretion of a significant amount of low-metallicity gas during the last significant merger $7 \mathrm{Gyr}$ ago is evident as a decrease in the metallicity of stars formed in situ immediately following this event. Comparing the different populations in the simulation, we note that the metallicities of halo stars formed in situ closely follow the evolution of disk stars, while the accreted halo is more metal-poor at all ages. The bifurcation in the metallicity tracks for the in situ and accreted halo is a prediction of the Latte simulation, which, if confirmed observationally, can be used to directly differentiate between the accreted and in situ halo stars.

\section{Discussion}

We have presented evidence that a substantial fraction the stellar halo in the solar neighborhood is metal-rich, and we now put this result in context of previous investigations (Section 5.1). Based on its chemical abundances and orbital properties, we infer that the metal-rich halo has been formed in situ, so we discuss mechanisms that heat stars originally formed in the disk to halo-like orbits (Section 5.2). We conclude the section by outlining a test of the in situ formation 

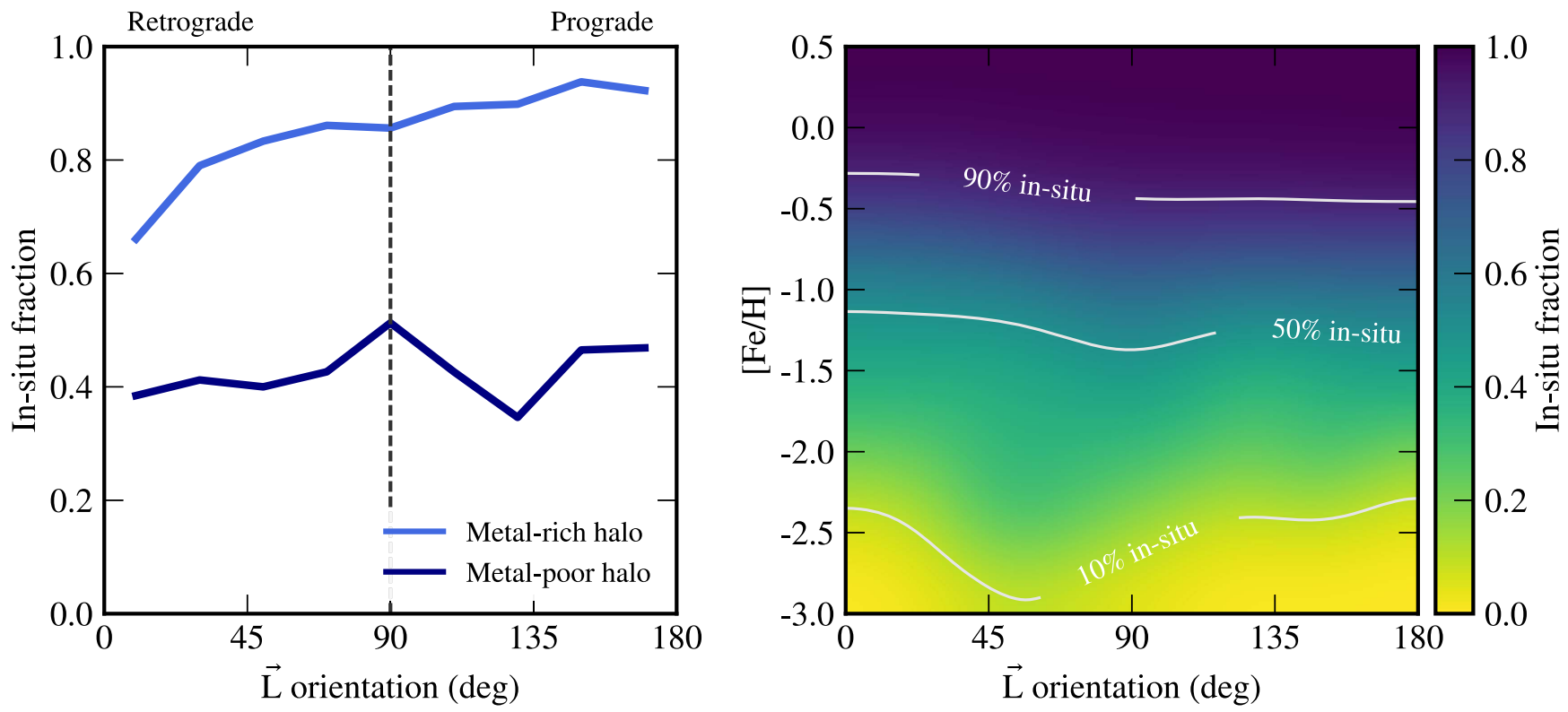

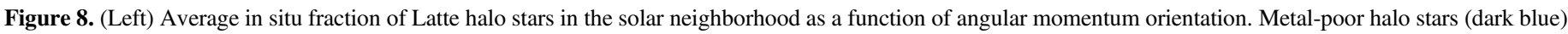

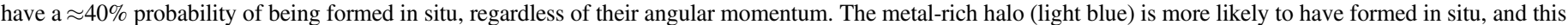

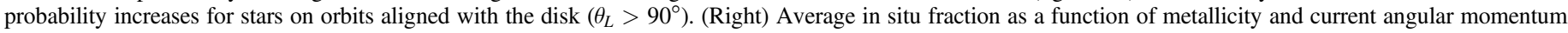

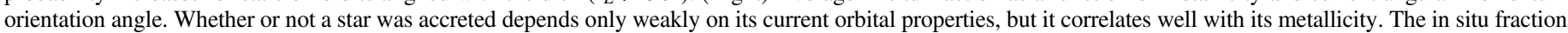

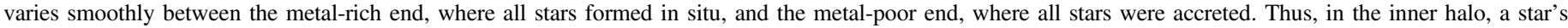
metallicity is a much better indicator of its origin (the probability that it was formed in situ) than its current kinematics.

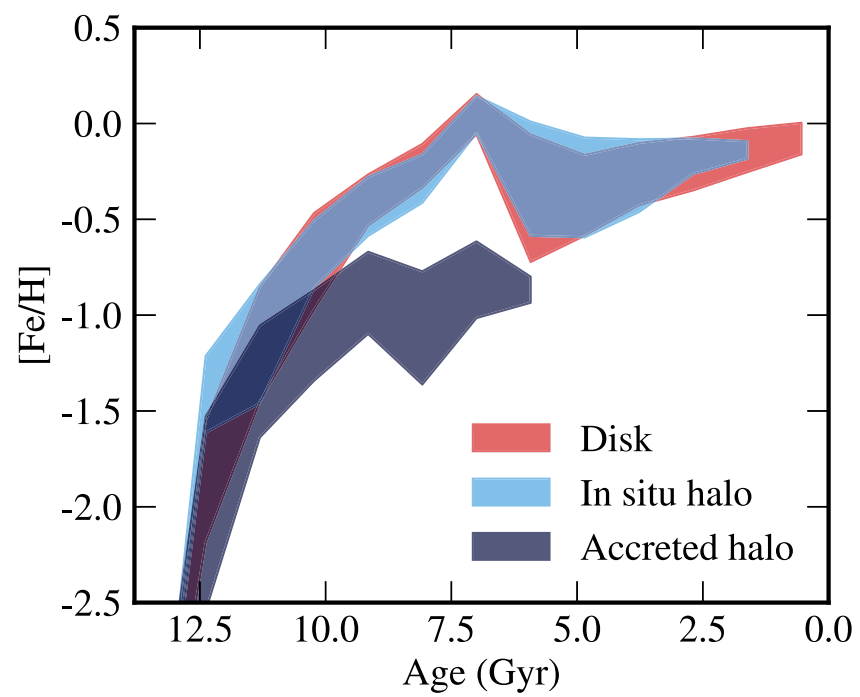

Figure 9. Metallicity range (16-84\%) of star particles in the Latte simulation, as a function of their age, for three structural components (defined as in Figures 6-7) identified at the solar circle: disk (red), in situ halo (light blue), and accreted halo (dark blue). The in situ halo follows the metallicity evolution of the disk, while accreted halo stars of the same age are consistently more metal-poor. This prediction can be directly tested once stellar ages are available for the Gaia stars.

scenario for the metal-rich halo component with stellar ages (Section 5.3).

\subsection{Previous Evidence for a Metal-Rich Halo}

Large-scale spectroscopic surveys have mapped the distribution of stellar metallicity in the Milky Way (e.g., Ivezić et al. 2008). Some of these stars have distance estimates, and can therefore be spatially identified with either the disk or the halo.
Relevant to this discussion, halo stars in the outer Galaxy, $R_{G}>15 \mathrm{kpc}$, are very metal-poor, with median $[\mathrm{Fe} / \mathrm{H}] \sim$ -2.2 , whereas the typical metallicity of a halo star in the inner Galaxy is $[\mathrm{Fe} / \mathrm{H}] \sim-1.6$ (e.g., Carollo et al. 2007; de Jong et al. 2010; Fernández-Alvar et al. 2017), which agrees well with the metal-poor halo component identified in our sample. Also similar to our findings, metallicities of the inner halo extend to the solar value, even though these are the tail of the metallicity distribution (e.g., Allende Prieto et al. 2006) and not an additional component that is visible in Figure 2.

However, employing only spatial information makes it hard to distinguish between the halo and the thick disk, especially at high metallicity. The difference between the thick disk and the halo is more pronounced in kinematics, so Sheffield et al. (2012) selected outliers from the disk velocity field to study the halo. They identified eight metal-rich halo stars, whose $\alpha$-abundances are consistent with the disk, and suggested these had been kicked out of the disk. Still, none of these stars have kinematics that could rule out a thick disk interpretation at a $3 \sigma$ level.

To date, only one metal-rich star is known to have a high probability of being a halo star (Hawkins et al. 2015). This star has high velocity in the Galactic rest frame, $V_{\mathrm{GSR}} \simeq 430 \mathrm{~km} \mathrm{~s}^{-1}$, and is on an eccentric orbit, $e=0.72$, that reaches $\sim 30 \mathrm{kpc}$ above the Galactic plane. However, it has a metallicity of $[\mathrm{Fe} / \mathrm{H}]=-0.18$, so Hawkins et al. (2015) concluded that it had been ejected from the thick disk. This star is a valuable indicator of the processes governing the assembly of the Galaxy. Being only a single star, however, it cannot establish how ubiquitous these processes are.

\subsection{Disk Heating Mechanisms}

The excess of metal-rich halo stars on prograde orbits indicates that they originate from within the Milky Way disk, 
but it is unclear at what Galactocentric distance these stars were formed. The bulk of similarly selected stars in Latte originated from the inner Galaxy, implying that a degree of radial migration and/or heating occurred. Alternatively, these stars could be runaway stars - stars kicked out of the disk at the solar radius during dynamical processes not captured within the Latte simulation. In this section, we explore implications of these opposing disk heating mechanisms, and assess how plausible they are in explaining metal-rich halo stars detected in RAVE-on-TGAS.

\subsubsection{Runaway Stars}

Runaway stars are young stars that were formed in the disk and ejected from their birthplace (Blaauw 1961). Some of them have been found in the halo (e.g., Conlon et al. 1990), so it is sensible to test whether any of our metal-rich halo stars are, in fact, runaways. Having recently formed in the disk, runaways should have high metallicities, and Bromley et al. (2009) have already suggested that solar-metallicity stars reported at $5 \mathrm{kpc}$ away from the Galactic place (Ivezić et al. 2008) could be runaway stars. The metallicity distribution of our metal-rich halo peaks at $[\mathrm{Fe} / \mathrm{H}] \approx-0.5$, so most of them are probably not runaway stars. However, the high-metallicity tail of our halo sample extends to super-solar values, so we test whether any of those stars are consistent with being runaways.

In addition to being metal-rich, runaway stars typically have an early spectral type. Therefore, to test the runaway origin of the metal-rich halo, we analyze the fraction of runaways expected in a disk population as a function of temperature. Due to difficulties in the spectroscopic analysis of hot stars, the RAVE-on catalog preferentially contains cold stars, with more than $80 \%$ of the sample being K stars. The expected fraction of runaway stars drops sharply with decreasing temperature, from $40 \%$ for $\mathrm{O}$ stars, $5 \%$ for B stars, and $\approx 2 \%$ for A stars, to negligible contributions from later stellar types (Blaauw 1961; Gies \& Bolton 1986; Bromley et al. 2009; Perets \& Šubr 2012). There are no OB stars in our halo sample, and only three A stars. These all have a super-solar metallicity, $[\mathrm{Fe} / \mathrm{H}]>0$, so they are prime candidates for runaway stars. However, they constitute only $0.5 \%$ of the metal-rich halo sample, so we can safely conclude that runaway stars are a minor component of the observed metal-rich stellar halo.

\subsubsection{Radial Migration}

In the classical picture of radial migration, spiral structures in the disk radially scatter nearby stars up to several kpc (Sellwood \& Binney 2002). Idealized simulations of disk formation have found radial migration to be a key component in explaining numerous observables, such as the spread in the age-metallicity relation (Roškar et al. 2008) or the disk morphology and its abundance patterns (Schönrich \& Binney 2009 b). Such studies have also shown that stars migrating outward reach larger heights above the disk plane (e.g., Schönrich \& Binney 2009b; Loebman et al. 2011). This scenario can explain, at least in part, the formation of the thick disk (e.g., Wilson et al. 2011), so it is also conceivable that the metal-rich stars we have identified on the halo-like orbits are an endpoint of this process. However, subsequent numerical works have reached the opposite conclusion: that outward migrators do not attain larger scale heights from the disk plane (Minchev et al. 2012; Vera-Ciro et al. 2014), thus casting doubts on the idea of forming the thick disk and a metal-rich halo through radial migration.

The above results on radial migration are based on simulations initialized to match the orderly morphology of stellar disks observed at the present day, and do not capture the range of dynamical conditions present in the cosmological simulations of galaxy formation (e.g., Agertz et al. 2009). Idealized simulations show that including density inhomogeneities in the gaseous disk heats up stellar disks (Struck \& Elmegreen 2017), as does allowing for interactions with massive satellites (Laporte et al. 2016). Such conditions were common at early cosmic times in the Latte simulation analyzed here. In particular, the metal-rich halo was formed before the formation of the thin disk (Ma et al. 2016b), while the host galaxy was still actively accreting $(z \gtrsim 1$, or age $\gtrsim 7 \mathrm{Gyr}$ ). Accretion from the inter-galactic medium and merging satellites brought a lot of gas into the galactic center (evident as star-forming tracks in Figure 7), which fueled additional in situ star formation. El-Badry et al. (2016) showed that these conditions create two mechanisms for radially displacing stars from their birth location. First, some stars are formed during gas outflows, so their initial orbits can be eccentric and have large apocenters. Second, the combination of inflowing gas accretion and gas outflows driven by stellar feedback produce strong fluctuations in the underlying gravitational potential. Such fluctuations have already been shown to change the distribution of dark matter, particularly in generating a cored density profile (e.g., Pontzen \& Governato 2012; Brooks \& Zolotov 2014; Di Cintio et al. 2014; Chan et al. 2015), but ElBadry et al. (2016) further showed that stellar orbits are affected as well, ultimately becoming heated to a more isotropic distribution. This mechanism is most efficient in relatively shallow potential wells of dwarf galaxies. Although the Milky Way-mass host galaxy in Latte is too massive to exhibit such behavior at $z \sim 0$, its progenitor had significantly lower stellar mass (was more gas rich) while the metal-rich halo was forming, so we suggest that a similar process drove the radial migration to the solar circle that we see here, consistent with conclusions of Ma et al. (2017). In future work, we will investigate this behavior in Latte in more detail.

Radial migration, driven by large-scale motions in the Milky Way progenitor, could explain the origin of metal-rich stars on halo-like orbits in the solar neighborhood. If these stars truly originate from the inner Galaxy, then they not only illustrate an important dynamical mechanism shaping the Galaxy, but are also a unique window into star formation in the early Milky Way. Future analysis and data from the Gaia mission will allow us to test this hypothesis, which we briefly discuss in the next section.

\subsection{Inferring the Formation Scenario of the Stellar Halo with Stellar Ages}

In section Section 4.3, we showed that the metal-rich halo simulated in Latte was chemically enriched in a fashion similar to the Latte disk. When compared at a fixed age, these components have higher metallicity than the metal-poor halo at all times (see Figure 9). We can directly test this prediction by dating the stars in our TGAS-RAVE-on sample. Unfortunately, stellar ages are not obtained easily (for a recent review, see Soderblom 2010). A number of observables that correlate with age have been identified, such as stellar rotation (Barnes 2007), chromospheric activity (Mamajek \& Hillenbrand 2008), and surface abundances (Ness et al. 2016), but none of these 
empirical relations are applicable to all of the field stars. Models of stellar evolution can relate the position of any star in the Hertzsprung-Russell diagram (HRD) and its internal structure to its age. The latter is inferred from asteroseismic studies of stellar pulsations, and has so far been employed to date a few dozen well observed stars (e.g., Silva Aguirre et al. 2015). In the coming decade, asteroseismic dating will be expanded, but still limited to the brightest stars (Rauer et al. 2014; Ricker et al. 2015). We expect the HRD age dating to be more easily applied to a larger sample of stars, and discuss it in more detail below.

Coeval stellar populations are routinely dated by comparison of their tracks in the HRD to theoretical isochrones (e.g., Sandage 1970; Chaboyer et al. 1998; Dotter et al. 2007), but isochrone dating of field stars is less straightforward. Intrinsically, without the HRD positions of coeval companions, age estimates of field stars are very uncertain in evolutionary stages that keep stars at an approximately constant position in the HRD, such as the main sequence phase. In addition, precisely measuring stellar distances (which are required to put a star on the HRD, as opposed to merely on a color-magnitude diagram) is observationally challenging. However, if distances are known, stellar ages can be measured for stars in pre- or post-main sequence evolutionary stages. Nordström et al. (2004) measured ages and other intrinsic stellar parameters for thousands of nearby field stars by obtaining their absolute magnitudes from Hipparcos parallaxes, effective temperatures, and metallicities from follow-up spectroscopy, and then reading off the age by interpolating theoretical isochrones in this three-dimensional space. TGAS has already increased the sample of stars with known distances by an order of magnitude, and several groups are modeling the multi-band stellar photometry (and including spectroscopy when available) to provide constraints on their ages. In such a procedure, ages of red giants are measured with a precision of 1-3 Gyr when only photometric data is available. Including spectroscopically derived stellar parameters reduces the uncertainty in recovered ages to $1 \mathrm{Gyr}$ (P. Cargile 2017, private communication). Most of the halo stars in our sample are giants, so if the bifurcation in the age-metallicity relation of halo stars exists at the level suggested by the simulation studied here, we will soon be able to detect it observationally.

\section{Summary}

The goal of this work was to investigate the origin of halo stars in the solar neighborhood. We analyzed a sample of stars located in 6D using the first year of Gaia data, combined with observations from ground-based spectroscopic surveys. The halo was defined by requiring a relative velocity with respect to the Local Standard of Rest larger than $220 \mathrm{~km} \mathrm{~s}^{-1}$. Metal-poor stars, $[\mathrm{Fe} / \mathrm{H}]<-1$, comprise approximately half of this kinematically defined halo sample. The other half of the sample are stars more metal-rich than expected for the inner halo $([\mathrm{Fe} / \mathrm{H}]>-1)$, and whose metallicity and $\alpha$-element abundances are traditionally associated with the disk population. We built a toy model of the solar neighborhood to show that orbits of the metal-poor halo stars are isotropically distributed, whereas the metal-rich halo is preferentially aligned with the Galactic disk. To uncover the origin of these two halo components, we performed an identical analysis on a sample of stars selected in a solar-like neighborhood of the Latte cosmological hydrodynamic simulation. A significant fraction of the simulated halo stars are also metal-rich and on prograde orbits with respect to the disk. Most of these metal-rich halo stars were formed in situ in the main galaxy. In general, we found that the origin of halo stars is well-correlated with stellar metallicity, with metal-poor stars having been accreted and metal-rich having formed in situ. However, the halo origin has little dependence on stellar kinematics. Additionally, the majority of metal-rich halo stars in Latte were formed in the inner galaxy and migrated several kpc outwards, indicating that radial redistribution actively operates in Milky Way-like galaxies. In this model, the in situ component of the stellar halo is more metal-rich than the accreted component at a fixed age, a hypothesis easily verifiable with forthcoming data.

We have presented for the first time a large population of metal-rich, $[\mathrm{Fe} / \mathrm{H}]>-1$, stars on halo-like orbits, and inferred that they have formed in situ. These conclusions arise in part from a remarkable agreement between the precise observational data from the Gaia mission and the Latte simulation of a Milky Way-like galaxy. Direct comparison of observed and simulated galaxies at this level of detail will be a powerful tool in studying the Galaxy in the Gaia era.

We thank the anonymous referee for carefully reviewing this manuscript and providing comments that improved its clarity. It is a pleasure to thank Andy Casey for providing a match of the RAVE-on catalog to TGAS, Yuan-Sen Ting for matching the APOGEE catalog to TGAS, as well as Kim Venn, Rosy Wyse, Warren Brown, and Elena D'Onghia for insightful comments that shaped the progression of this project.

This work has made use of the following Python packages: matplotlib (Hunter 2007), numpy (Walt et al. 2011), scipy (Jones et al. 2001), Astropy (Astropy Collaboration et al. 2013), and gala (Price-Whelan et al. 2017).

This paper was written in part at the 2016 NYC Gaia Sprint, hosted by the Center for Computational Astrophysics at the Simons Foundation in New York City.

A.B. was supported by an Institute for Theory and Computation Fellowship. C.C. acknowledges support from the Packard Foundation. A.W. was supported by a Caltech-Carnegie Fellowship, in part through the Moore Center for Theoretical Cosmology and Physics at Caltech, and by NASA through grant HST-GO-14734 from STScI. D.K. was supported by NSF grant AST-1412153 and a Cottrell Scholar Award from the Research Corporation for Science Advancement.

This work has made use of data from the European Space Agency (ESA) mission Gaia (http://www.cosmos.esa.int/ gaia), processed by the Gaia Data Processing and Analysis Consortium (DPAC, http://www.cosmos.esa.int/web/gaia/ dpac/consortium). Funding for the DPAC has been provided by national institutions, particularly the institutions participating in the Gaia Multilateral Agreement.

Funding for RAVE has been provided by: the Australian Astronomical Observatory; the Leibniz-Institut fuer Astrophysik Potsdam (AIP), the Australian National University; the Australian Research Council; the French National Research Agency; the German Research Foundation (SPP 1177 and SFB 881); the European Research Council (ERC-StG 240271 Galactica); the Istituto Nazionale di Astrofisica at Padova; Johns Hopkins University; the National Science Foundation of the USA (AST0908326); the W.M. Keck foundation; Macquarie University; the Netherlands Research School for Astronomy; the Natural Sciences and Engineering Research Council of Canada; the Slovenian 


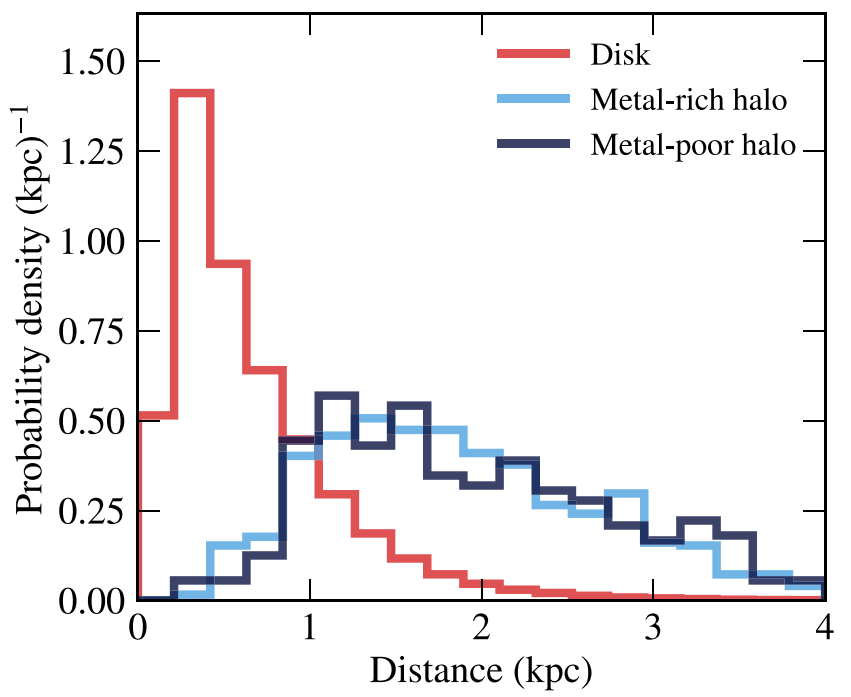

Figure 10. Distribution of heliocentric distances in the TGAS-RAVE-on sample, split by kinematically identified components. The disk stars (red) are generally closer than the halo (blue), but we see no difference in the distances of the metal-rich (light blue) and metal-poor halo components (dark blue). Orbital differences observed between the metal-rich and the metal-poor halo do not originate from a difference in their spatial distributions.

Research Agency; the Swiss National Science Foundation; the Science \& Technology Facilities Council of the UK; Opticon; Strasbourg Observatory; and the Universities of Groningen, Heidelberg, and Sydney. The RAVE web site is at https://www. rave-survey.org.

Funding for the Sloan Digital Sky Survey IV has been provided by the Alfred P. Sloan Foundation, the U.S. Department of Energy Office of Science, and the participating institutions. SDSS-IV acknowledges support and resources from the Center for HighPerformance Computing at the University of Utah. The SDSS web site is www.sdss.org.

SDSS-IV is managed by the Astrophysical Research Consortium for the Participating Institutions of the SDSS Collaboration, including: the Brazilian Participation Group, the Carnegie Institution for Science, Carnegie Mellon University, the Chilean Participation Group, the French Participation Group, the HarvardSmithsonian Center for Astrophysics, Instituto de Astrofísica de Canarias, Johns Hopkins University, the Kavli Institute for the Physics and Mathematics of the Universe (IPMU)/University of Tokyo, Lawrence Berkeley National Laboratory, Leibniz Institut für Astrophysik Potsdam (AIP), Max-Planck-Institut für Astronomie (MPIA Heidelberg), Max-Planck-Institut für Astrophysik (MPA Garching), Max-Planck-Institut für Extraterrestrische Physik (MPE), National Astronomical Observatories of China, New Mexico State University, New York University, the University of Notre Dame, Observatário Nacional/MCTI, The Ohio State University, Pennsylvania State University, Shanghai Astronomical Observatory, the United Kingdom Participation Group, Universidad Nacional Autónoma de México, University of Arizona, University of Colorado Boulder, University of Oxford, University of Portsmouth, University of Utah, University of Virginia, University of Washington, University of Wisconsin, Vanderbilt University, and Yale University.

\section{Appendix A}

\section{Distribution of Distances in the TGAS-RAVE-on Sample}

The TGAS-RAVE-on sample employed in this work is exploring the solar neighborhood, but is not volume-complete.
To illustrate the spatial extent of the sample, we show the distribution of heliocentric distances in Figure 10, and compare the distances of the kinematically selected components defined in Section 3.1. This sample is local, in the sense that more than $90 \%$ of the stars are within $1.5 \mathrm{kpc}$ from the Sun. As expected from our position close to the Galactic plane, the disk stars (red line) are preferentially closer than the halo stars (blue lines). Ninety percent of the disk is contained within $1.3 \mathrm{kpc}$, whereas the 90th percentile in the halo distance extends to $3 \mathrm{kpc}$. On the other hand, the two halo components split by metallicity have a similar distribution of distances and are spatially indistinguishable within this volume.

\section{Appendix B Thick-disk Contamination}

The thick disk bridges the thin disk and the halo in both chemical abundances and kinematics. Given that the metal-rich halo identified in this study has abundances consistent with the thick disk, here we quantify how different it is from the canonical thick disk kinematically.

As demonstrated by the toy model of the solar neighborhood (Section 4.1), we expect some thick disk stars to enter our halo selection (Figure 5, red points above the thick black line in the top panel). We visualize the expected contamination levels in the left panel of Figure 11 by drawing the probability contours for the thick disk velocity ellipsoid (Bensby et al. 2003) in the Toomre diagram. The successive contours enclose the parameter space occupied by the thick disk with probabilities of $68 \%, 95 \%, 99.7 \%$, and $99.9 \%$ (labeled as $1-4 \sigma$ in Figure 11). Halo stars from our sample are shown as points, with metal-rich being represented by light blue circles and metal-poor by dark blue squares. All of the halo stars are outside the $3 \sigma$ thick disk contour, and thus inconsistent with being a thick disk at the $99.7 \%$ level, but $145(\approx 25 \%)$ metalrich and $25(\approx 7 \%)$ metal-poor halo stars are inside the $4 \sigma$ contour. On the other hand, there are 453 metal-rich halo stars outside the $4 \sigma$ thick disk contour, whereas the toy model expects only 16 thick disk stars in this region. Even though the velocity distributions of the halo and the thick disk overlap, and our halo sample may not be completely free of thick-disk contaminants, there is a clear excess of stars with thick-disk abundances beyond the canonical thick-disk kinematics.

In the right panel of Figure 11, we quantify the probability of halo stars in our sample being a part of the thick disk, fully accounting for the observational uncertainties in all six observables. The light blue line shows the cumulative fraction of metal-rich halo stars being a thick disk star at a given probability, while dark blue is the corresponding line for the metal-poor halo stars. Less than $20 \%$ of metal-rich halo stars have more than a $1 \%$ probability (marked by a vertical black line) of being a misclassified thick disk star. For the metal-poor halo, this fraction is even lower, at $5 \%$. The median probability of being a thick disk star is $\sim 3 \times 10^{-4}$ and $\sim 2 \times 10^{-6}$ for the metal-rich and the metal-poor halo, respectively, ruling out the thick disk interpretation of metal-rich stars identified in the local stellar halo.

Thus far, we have only considered the kinematic definition of a thick disk as measured by Bensby et al. (2003). Studies based on different samples have arrived at slightly modified properties of a thick-disk velocity ellipsoid (e.g., Soubiran et al. 2003; Carollo et al. 2010; Kordopatis et al. 2013). Furthermore, in a theoretical study of a thick disk formed in an idealized 

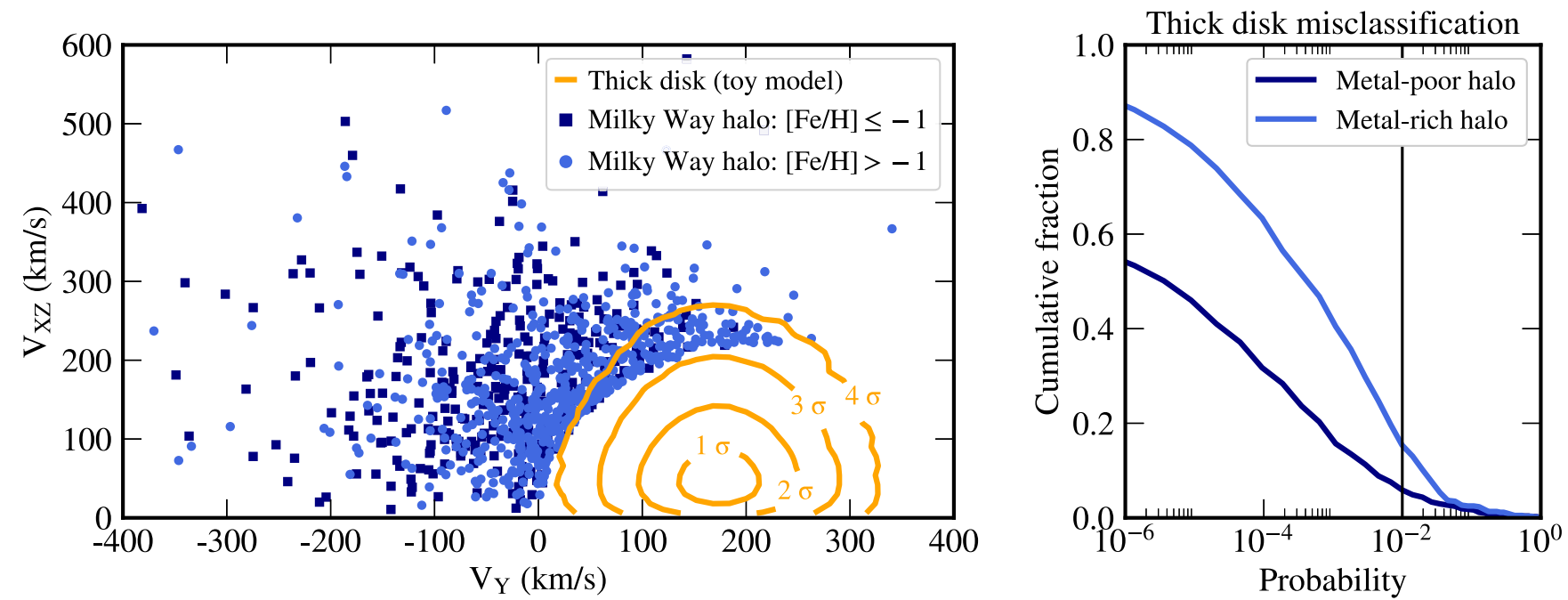

Figure 11. (Left) Probability contours of toy-model thick-disk stars in the Toomre diagram, in whole steps of standard deviation, $\sigma$ (orange lines). All halo stars from our RAVE-on-TGAS sample (metal-rich in light blue circles and metal-poor in dark blue squares) lie outside the $3 \sigma$ thick disk contour, but some are consistent with the thick disk at a $4 \sigma$ level. (Right) Probability for stars, identified in RAVE-on-TGAS as part of the halo, of actually being a part of the thick disk. Lines show cumulative fractions of halo stars as a function of this probability, with light blue for the metal-rich and dark blue for the metal-poor halo stars. Only a small fraction of both halo components is expected to be a misclassified part of the thick disk (20\% of the metal-rich and $5 \%$ of the metal-poor halo have a thick-disk probability larger than $1 \%$, marked with a black vertical line).

simulation, Schönrich \& Binney (2009a) noted that its velocity distribution in the Toomre diagram is much more asymmetric than the usually assumed Gaussian distribution function. However, even this more extended definition of a thick disk does not encompass all of the metal-rich stars identified in the solar neighborhood, particularly excluding stars on retrograde orbits with high $V_{X Z}$. Assuming a different distribution function for the thick disk changes the inferred contamination levels in our halo sample in detail, but no disk-like distribution explains stars on very retrograde, kinematically hot orbits, where some of the metal-rich stars from our sample are found.

\section{ORCID iDs}

Ana Bonaca (i) https://orcid.org/0000-0002-7846-9787 Charlie Conroy (1) https://orcid.org/0000-0002-1590-8551 Andrew Wetzel (1) https://orcid.org/0000-0003-0603-8942 Philip F. Hopkins (다 https://orcid.org/0000-0003-3729-1684 Dušan Kereš (i) https://orcid.org/0000-0002-1666-7067

\section{References}

Agertz, O., Teyssier, R., \& Moore, B. 2009, MNRAS, 397, L64 Alam, S., Albareti, F. D., Allende Prieto, C., et al. 2015, ApJS, 219, 12 Allende Prieto, C., Beers, T. C., Wilhelm, R., et al. 2006, ApJ, 636, 804 Astropy Collaboration, Robitaille, T. P., Tollerud, E. J., et al. 2013, A\&A, 558, A33

Barnes, S. A. 2007, ApJ, 669, 1167

Beers, T. C., Carollo, D., Ivezić, Ž, et al. 2012, ApJ, 746, 34 Bell, E. F., Zucker, D. B., Belokurov, V., et al. 2008, ApJ, 680, 295 Belokurov, V., Evans, N. W., Bell, E. F., et al. 2007a, ApJL, 657, L89 Belokurov, V., Evans, N. W., Irwin, M. J., et al. 2007b, ApJ, 658, 337 Bensby, T., Feltzing, S., \& Lundström, I. 2003, A\&A, 410, 527

Bernard, E. J., Ferguson, A. M. N., Schlafly, E. F., et al. 2016, MNRAS, 463, 1759

Binney, J., \& Tremaine, S. 2008, Galactic Dynamics (2nd ed.; Princeton, NJ: Princeton Univ. Press)

Blaauw, A. 1961, BAN, 15, 265

Bland-Hawthorn, J., \& Gerhard, O. 2016, ARA\&A, 54, 529

Bonaca, A., Geha, M., \& Kallivayalil, N. 2012a, ApJL, 760, L6

Bonaca, A., Jurić, M., Ivezić, Ž, et al. 2012b, AJ, 143, 105

Bromley, B. C., Kenyon, S. J., Brown, W. R., \& Geller, M. J. 2009, ApJ, 706, 925
Brooks, A. M., \& Zolotov, A. 2014, ApJ, 786, 87

Bullock, J. S., \& Johnston, K. V. 2005, ApJ, 635, 931

Carollo, D., Beers, T. C., Chiba, M., et al. 2010, ApJ, 712, 692

Carollo, D., Beers, T. C., Lee, Y. S., et al. 2007, Natur, 450, 1020

Casey, A. R., Hawkins, K., Hogg, D. W., et al. 2017, ApJ, 840, 59

Chaboyer, B., Demarque, P., Kernan, P. J., \& Krauss, L. M. 1998, ApJ, 494, 96 Chan, T. K., Kereš, D., Oñorbe, J., et al. 2015, MNRAS, 454, 2981

Conlon, E. S., Dufton, P. L., Keenan, F. P., \& Leonard, P. J. T. 1990, A\&A, 236, 357

Cook, B. A., Conroy, C., Pillepich, A., Rodriguez-Gomez, V., \& Hernquist, L. 2016, ApJ, 833, 158

Cooper, A. P., Cole, S., Frenk, C. S., et al. 2010, MNRAS, 406, 744

Cooper, A. P., Parry, O. H., Lowing, B., Cole, S., \& Frenk, C. 2015, MNRAS, 454, 3185

Crnojević, D., Sand, D. J., Spekkens, K., et al. 2016, ApJ, 823, 19

Deason, A. J., Belokurov, V., Evans, N. W., \& Johnston, K. V. 2013, ApJ, 763,113

Deason, A. J., Belokurov, V., Koposov, S. E., et al. 2017, arXiv:1703.09230 de Jong, J. T. A., Yanny, B., Rix, H.-W., et al. 2010, ApJ, 714, 663 Di Cintio, A., Brook, C. B., Macciò, A. V., et al. 2014, MNRAS, 437, 415

Diemand, J., Kuhlen, M., Madau, P., et al. 2008, Natur, 454, 735

Diemer, B., \& Kravtsov, A. V. 2014, ApJ, 789, 1

Dotter, A., Chaboyer, B., Jevremović, D., et al. 2007, AJ, 134, 376

Eggen, O. J., Lynden-Bell, D., \& Sandage, A. R. 1962, ApJ, 136, 748

El-Badry, K., Wetzel, A., Geha, M., et al. 2016, ApJ, 820, 131

ESA (ed.) 1997, in HIPPARCOS and TYCHO Catalogues, ESA SP Series Vol. 1200 (Noordwijk: ESA)

Feldmann, R., Quataert, E., Hopkins, P. F., Faucher-Giguère, C.-A., \& Kereš, D. 2016, arXiv:1610.02411

Ferguson, A. M. N., Irwin, M. J., Ibata, R. A., Lewis, G. F., \& Tanvir, N. R. 2002, AJ, 124, 1452

Fernández-Alvar, E., Carigi, L., Allende Prieto, C., et al. 2017, MNRAS, 465,1586

Font, A. S., McCarthy, I. G., Crain, R. A., et al. 2011, MNRAS, 416, 2802

Gaia Collaboration, Brown, A. G. A., Vallenari, A., et al. 2016a, A\&A, 595, A2 Gaia Collaboration, Prusti, T., de Bruijne, J. H. J., et al. 2016b, A\&A, 595, A1 Gies, D. R., \& Bolton, C. T. 1986, ApJS, 61, 419

Gilmore, G., Wyse, R. F. G., \& Kuijken, K. 1989, ARA\&A, 27, 555

Grillmair, C. J. 2009, ApJ, 693, 1118

Grillmair, C. J., \& Carlin, J. L. 2016, ASSL, 420, 87

Grillmair, C. J., \& Dionatos, O. 2006, ApJL, 643, L17

Hawkins, K., Kordopatis, G., Gilmore, G., et al. 2015, MNRAS, 447, 2046

Helmi, A., Veljanoski, J., Breddels, M. A., Tian, H., \& Sales, L. V. 2017, A\&A, 598, A58

Helmi, A., \& White, S. D. M. 1999, MNRAS, 307, 495

Helmi, A., White, S. D. M., de Zeeuw, P. T., \& Zhao, H. 1999, Natur, 402, 53

Høg, E., Fabricius, C., Makarov, V. V., et al. 2000, A\&A, 355, L27 
Holtzman, J. A., Shetrone, M., Johnson, J. A., et al. 2015, AJ, 150, 148 Hopkins, P. F. 2015, MNRAS, 450, 53

Hopkins, P. F., Keres, D., Onorbe, J., et al. 2014, MNRAS, 445, 581

Hopkins, P. F., Wetzel, A., Keres, D., et al. 2017, arXiv:1702.06148

Hunter, J. D. 2007, CSE, 9, 90

Ibata, R., Irwin, M., Lewis, G., Ferguson, A. M. N., \& Tanvir, N. 2001, Natur, 412, 49

Ibata, R. A., Gilmore, G., \& Irwin, M. J. 1994, Natur, 370, 194

Ivezić, Ž, Sesar, B., Jurić, M., et al. 2008, ApJ, 684, 287

Janesh, W., Morrison, H. L., Ma, Z., et al. 2016, ApJ, 816, 80

Jones, E., Oliphant, T., Peterson, P., et al. 2001, SciPy: Open Source Scientific Tools for Python, http://www.scipy.org/

Jurić, M., Ivezić, Ž, Brooks, A., et al. 2008, ApJ, 673, 864

Kafle, P. R., Sharma, S., Robotham, A. S. G., et al. 2017, arXiv:1706.02301

Klypin, A. A., Trujillo-Gomez, S., \& Primack, J. 2011, ApJ, 740, 102

Kordopatis, G., Gilmore, G., Wyse, R. F. G., et al. 2013, MNRAS, 436, 3231

Kunder, A., Kordopatis, G., Steinmetz, M., et al. 2017, AJ, 153, 75

Laporte, C. F. P., Gómez, F. A., Besla, G., Johnston, K. V., \& Garavito-Camargo, N. 2016, arXiv:1608.04743

Lee, D. M., Johnston, K. V., Sen, B., \& Jessop, W. 2015, ApJ, 802, 48

Lindegren, L., Lammers, U., Bastian, U., et al. 2016, A\&A, 595, A4

Loebman, S. R., Roškar, R., Debattista, V. P., et al. 2011, ApJ, 737, 8

Ma, X., Hopkins, P. F., Faucher-Giguère, C.-A., et al. 2016a, MNRAS, 456, 2140

Ma, X., Hopkins, P. F., Wetzel, A. R., et al. 2016b, arXiv:1608.04133

Ma, X., Hopkins, P. F., Wetzel, A. R., et al. 2017, MNRAS, 467, 2430

Majewski, S. R., Schiavon, R. P., Frinchaboy, P. M., et al. 2015, arXiv:1509.05420

Mamajek, E. E., \& Hillenbrand, L. A. 2008, ApJ, 687, 1264

Martínez-Delgado, D., Gabany, R. J., Crawford, K., et al. 2010, AJ, 140, 962

Martínez-Delgado, D., Romanowsky, A. J., Gabany, R. J., et al. 2012, ApJL, 748, L24

Michalik, D., Lindegren, L., \& Hobbs, D. 2015, A\&A, 574, A115

Minchev, I., Famaey, B., Quillen, A. C., et al. 2012, A\&A, 548, A127

Ness, M., Hogg, D. W., Rix, H.-W., et al. 2016, ApJ, 823, 114

Ness, M., Hogg, D. W., Rix, H.-W., Ho, A. Y. Q., \& Zasowski, G. 2015, ApJ, 808, 16

Nidever, D. L., Bovy, J., Bird, J. C., et al. 2014, ApJ, 796, 38

Nissen, P. E., \& Schuster, W. J. 2010, A\&A, 511, L10

Nordström, B., Mayor, M., Andersen, J., et al. 2004, A\&A, 418, 989

Perets, H. B., \& Šubr, L. 2012, ApJ, 751, 133

Perryman, M. A. C., de Boer, K. S., Gilmore, G., et al. 2001, A\&A, 369, 339

Pontzen, A., \& Governato, F. 2012, MNRAS, 421, 3464

Price-Whelan, A., Sipocz, B., \& Oh, S. 2017, adrn/gala, v0.1.3 Zenodo, doi:10.5281/zenodo.321907
Price-Whelan, A. M., Johnston, K. V., Valluri, M., et al. 2016, MNRAS, 455, 1079

Purcell, C. W., Bullock, J. S., \& Kazantzidis, S. 2010, MNRAS, 404, 1711

Rauer, H., Catala, C., Aerts, C., et al. 2014, ExA, 38, 249

Ricker, G. R., Winn, J. N., Vanderspek, R., et al. 2015, JATIS, 1, 014003

Rockosi, C. M., Odenkirchen, M., Grebel, E. K., et al. 2002, AJ, 124, 349

Romanowsky, A. J., Strader, J., Brodie, J. P., et al. 2012, ApJ, 748, 29

Roškar, R., Debattista, V. P., Quinn, T. R., Stinson, G. S., \& Wadsley, J. 2008, ApJL, 684, L79

Samland, M., \& Gerhard, O. E. 2003, A\&A, 399, 961

Sandage, A. 1970, ApJ, 162, 841

Sanderson, R. E., Helmi, A., \& Hogg, D. W. 2015, ApJ, 801, 98

Schlaufman, K. C., Rockosi, C. M., Lee, Y. S., et al. 2012, ApJ, 749, 77

Schönrich, R., Asplund, M., \& Casagrande, L. 2011, MNRAS, 415, 3807

Schönrich, R., Asplund, M., \& Casagrande, L. 2014, ApJ, 786, 7

Schönrich, R., \& Binney, J. 2009a, MNRAS, 399, 1145

Schönrich, R., \& Binney, J. 2009b, MNRAS, 396, 203

SDSS Collaboration, Albareti, F. D., Allende Prieto, C., et al. 2016 , arXiv: 1608.02013

Searle, L., \& Zinn, R. 1978, ApJ, 225, 357

Sellwood, J. A., \& Binney, J. J. 2002, MNRAS, 336, 785

Sheffield, A. A., Majewski, S. R., Johnston, K. V., et al. 2012, ApJ, 761, 161

Silva Aguirre, V., Davies, G. R., Basu, S., et al. 2015, MNRAS, 452, 2127

Smith, M. C., Evans, N. W., Belokurov, V., et al. 2009, MNRAS, 399, 1223

Soderblom, D. R. 2010, ARA\&A, 48, 581

Soubiran, C., Bienaymé, O., \& Siebert, A. 2003, A\&A, 398, 141

Sparre, M., Hayward, C. C., Feldmann, R., et al. 2015, MNRAS, 466, 88

Springel, V., Wang, J., Vogelsberger, M., et al. 2008, MNRAS, 391, 1685

Steinmetz, M., Zwitter, T., Siebert, A., et al. 2006, AJ, 132, 1645

Struck, C., \& Elmegreen, B. G. 2017, MNRAS, 469, 1157

Su, K.-Y., Hopkins, P. F., Hayward, C. C., et al. 2016, arXiv:1607.05274 van Leeuwen, F. 2007, A\&A, 474, 653

Venn, K. A., Irwin, M., Shetrone, M. D., et al. 2004, AJ, 128, 1177

Vera-Ciro, C., D'Onghia, E., Navarro, J., \& Abadi, M. 2014, ApJ, 794, 173

Walt, S. V. D., Colbert, S. C., \& Varoquaux, G. 2011, CSE, 13, 22

Watson, F. G., Parker, Q. A., Bogatu, G., et al. 2000, Proc. SPIE, 4008, 123

Wetzel, A. R., Hopkins, P. F., Kim, J.-h., et al. 2016, ApJL, 827, L23

Wheeler, C., Oñorbe, J., Bullock, J. S., et al. 2015, MNRAS, 453, 1305

White, S. D. M., \& Rees, M. J. 1978, MNRAS, 183, 341

Wilson, M. L., Helmi, A., Morrison, H. L., et al. 2011, MNRAS, 413, 2235

Wojno, J., Kordopatis, G., Piffl, T., et al. 2016, arXiv:1611.00733

Zasowski, G., Johnson, J. A., Frinchaboy, P. M., et al. 2013, AJ, 146, 81

Zolotov, A., Willman, B., Brooks, A. M., et al. 2009, ApJ, 702, 1058 